\title{
Eigenstates and transmission coefficients of finite-sized carbon nanotubes
}

\author{
S. Compernolle, L. Chibotaru, and A. Ceulemans \\ Department of Chemistry, University of Leuven, Celestijnenlaan 200F, B-3001 Leuven, Belgium
}

(Received 12 March 2003; accepted 8 May 2003)

\begin{abstract}
The tight-binding eigenstates of isolated finite-sized zigzag and armchair nanotubes are obtained analytically using the transfer matrix method. Edge states are encountered for zigzag tubes but not for armchair tubes. Inclusion of curvature leads to a decaying-oscillating behavior of the highest occupied and lowest unoccupied molecular orbital gap in armchair tubes as a function of length. For zigzag tubes the inclusion of curvature induces the conversion of an extended state at nonbonding energy to an edge state. In addition the transmission coefficient of zigzag and armchair tubes with featureless leads is analytically obtained. The existence of a transmission peak at the Fermi level that decays exponentially with nanotube length for zigzag tubes is explained. (C) 2003 American Institute of Physics. [DOI: 10.1063/1.1587691]
\end{abstract}

\section{INTRODUCTION}

Single-wall carbon nanotubes ${ }^{1}$ continue to be the subject of intense theoretical and experimental investigation, in view of their interesting electric properties as quasi-onedimensional (quasi-1D) structures. Depending on helicity, these tubes can occur both as metals and as semiconductors. The effect of, e.g., junctions, defects, curvature, and finite size on the electronic properties is reviewed in Ref. 2. The finite-size influence on the local density of states (LDOS) was studied by Jishi et al. ${ }^{3}$ The theoretical scanningtunneling-spectroscopy image of finite armchair tubes was generated by Rubio et al. ${ }^{4}$ and explained using a onedimensional quantum box model. Li et al. ${ }^{5}$ use a combination of a calculation for a 2D periodic solid (i.e., graphite) and a selection of wave functions due to the boundary conditions for a finite armchair tube to obtain a theoretical simulation of the highest occupied molecular orbital (HOMO) and the lowest unoccupied molecular orbital (LUMO) wave functions. Boundary effects on the band structure of ribbons, which can be considered as nanotubes with infinite diameter, were investigated by Klein ${ }^{6}$ and Fujita and co-workers. ${ }^{7-9}$ Ribbons with zigzag edges have edge states while ribbons with armchair edges have not. Similar conclusions for tubes have been obtained by Treboux et al. ${ }^{10}$ The electronic structure of finite-sized armchair nanotubes was investigated by Rochefort et al. ${ }^{11}$ and Sato and co-workers. ${ }^{12,13}$ They found an oscillating HOMO-LUMO gap with nanotube length. A more general investigation of band-gap oscillations is found in Refs. 14 and 15. The authors of Ref. 14 show the limitations of a simple "particle-in-a-box" model and propose instead the presence or absence of an alternating double-bond configuration to explain the band-gap oscillation.

Orlikowski and co-workers ${ }^{16}$ investigated the transmission properties of finite-sized nanotubes coupled to featureless leads numerically within a tight-binding frame. They obtained a peaked conductance spectrum that resembles the LDOS of the tube. For zigzag tubes, they find a transmission peak at the Fermi level that decays with length. For armchair tubes, they distinguish on- and off-resonance devices, de- pending on whether the number of rings of the tube is equal to $3 q-1$ or not.

In this work we apply the transfer matrix approach, ${ }^{17,18}$ which is a universal method for systems with tridiagonal block Hamiltonians. It yields analytical expressions for the eigenstates and transmission coefficients of the finite-sized zigzag and armchair nanotubes. Zigzag tubes can be terminated with hydrogen ends $(\mathrm{H})$ or with methylene ends $(\mathrm{M})$. We find that tubes with one hydrogen end and one methylene end have a twice as large HOMO-LUMO gap as zigzag tubes with identical ends and comparable length. The inclusion of curvature in the system converts an extended state at energy zero to an edge state. Due to these edge states, there is a transmission peak at the Fermi level that decays with nanotube length. Finally, we obtain that the high density of states around $E= \pm \beta_{1}$ for zigzag tubes does not add to the transmission around these energies. The inclusion of curvature is of fundamental importance for the description of the HOMO-LUMO gap oscillation with length of armchair tubes. Curvature effects also affect the distinction between on- and off-resonance devices.

\section{METHOD}

\section{A. General linear chain model}

The transfer matrix method is a general mathematical tool for systems that can be described by a tridiagonal block Hamiltonian. It constitutes a universal method for the study of quasi-1D systems. The Schrödinger equation for such systems has the following general form:

$$
E \mathbf{c}_{n}=L_{n-1}^{\dagger} \mathbf{c}_{n-1}+H_{n} \mathbf{c}_{n}+L_{n} \mathbf{c}_{n+1},
$$

where $H_{n}$ and $L_{n}$ are complex square matrices of size $M$ $\times M$, with the requirement $H_{n}=H_{n}^{\dagger}$ and $\operatorname{det}\left(L_{n}\right) \neq 0$. The matrix $H_{n}$ describes the internal dynamics of section $n$, and the matrix $L_{n}$ couples the adjacent sections $n$ and $n+1$. The vector $\mathbf{c}_{n}$ is of length $M$ and contains the coefficients of the orbitals in section $n$. The Hamiltonian $\mathcal{H}$ of the total system 
is a tridiagonal block Hamiltonian with diagonal blocks $H_{n}$ and upper and lower adjacent blocks $L_{n}$ and $L_{n}^{\dagger}$. Equation (1) can be written in matrix form:

$$
\begin{aligned}
& \left(\begin{array}{c}
\mathbf{c}_{n+1} \\
\mathbf{c}_{n}
\end{array}\right)=T_{n}\left(\begin{array}{c}
\mathbf{c}_{n} \\
\mathbf{c}_{n-1}
\end{array}\right), \\
& T_{n}=\left(\begin{array}{cc}
L_{n}^{-1}\left(E I-H_{n}\right) & -L_{n}^{-1} L_{n-1}^{\dagger} \\
I & 0
\end{array}\right),
\end{aligned}
$$

where we introduced the one-step transfer matrix $T_{n}$ with size $2 M \times 2 M$. In our case we consider a "chain" of finite length $N$ which corresponds to the boundary conditions $\mathbf{c}_{0}$ $=\mathbf{c}_{N+1}=0$. We set $L_{0}=L_{N}=I$. The following relation between $\mathbf{c}_{N}$ and $\mathbf{c}_{1}$ exists:

$$
\left(\begin{array}{c}
0 \\
\mathbf{c}_{N}
\end{array}\right)=T\left(\begin{array}{c}
\mathbf{c}_{1} \\
0
\end{array}\right), \quad T=T_{N} \cdots T_{1}=\left(\begin{array}{cc}
T_{11} & T_{12} \\
T_{21} & T_{22}
\end{array}\right) .
$$

The matrix $T$ is called the transfer matrix. The eigenvalues of $\mathcal{H}$ can now be found using the following relationship: ${ }^{17}$

$$
\operatorname{det}\left(T_{11}(E)\right)=\operatorname{det}\left(L_{1} \cdots L_{N-1}\right)^{-1} \operatorname{det}(E I-\mathcal{H}),
$$

which implies we have to solve $\operatorname{det}\left(T_{11}(E)\right)=0$.

When the chain is placed between two leads the Hamiltonian $\mathcal{H}$ undergoes the following change: ${ }^{19}$

$$
\mathcal{H} \Rightarrow \mathcal{H}+\Sigma, \quad \Sigma=\Sigma_{L}+\Sigma_{R}
$$

with

$$
\Sigma_{L}=\tau_{L}^{\dagger} G_{L} \tau_{L}, \quad \Sigma_{R}=\tau_{R}^{\dagger} G_{R} \tau_{R} .
$$

The matrices $\Sigma_{L}, \Sigma_{R}$ are retarded self-energies due to the left and right leads, respectively, which incorporate the effect of half-infinite leads on the chain. They can be calculated with the retarded Green's function of the half-infinite leads, $G_{R}$ and $G_{L}$,

$$
\begin{aligned}
G_{R} & =\left[\left(E+i 0^{+}\right) I-\mathcal{H}_{R}\right]^{-1}, \\
G_{L} & =\left[\left(E+i 0^{+}\right) I-\mathcal{H}_{L}\right]^{-1},
\end{aligned}
$$

and the matrices $\tau_{L}$ and $\tau_{R}$ connecting the chain to the halfinfinite left and right lead, respectively; $\mathcal{H}_{L}$ and $\mathcal{H}_{R}$ are the Hamiltonians of the isolated left and right leads, respectively. The self-energies are complex symmetric matrices in general:

$$
\Sigma_{L}=\Lambda_{L}-i \Delta_{L}, \quad \Sigma_{R}=\Lambda_{R}-i \Delta_{R},
$$

where $\Lambda$ and $\Delta$ are real symmetric matrices and the diagonal elements of $\Delta$ are positive by this definition. If the leads only interact with the edges of the chain, only the matrix blocks $H_{1}$ and $H_{N}$ will be affected:

$$
H_{1} \Rightarrow H_{1}+\Sigma_{11}, \quad H_{N} \Rightarrow H_{N}+\Sigma_{N N},
$$

with $\Sigma_{11}$ the-only non zero-matrix block of $\Sigma_{L}$ at position 11 and $\Sigma_{N N}$ the-only nonzero-matrix block of $\Sigma_{R}$ at position $N N$. So the transfer matrix changes only in its factors $T_{1}$ and $T_{N}$.

\section{B. Calculation of transmission}

The transmission can be calculated with the well-known formula $^{20}$

$$
\mathcal{T}=\operatorname{Tr}\left(\Gamma_{L} G \Gamma_{R} G^{\dagger}\right),
$$

with

$$
\begin{aligned}
& G=(E I-\mathcal{H}-\Sigma)^{-1}, \\
& \Gamma_{L}=-2 \operatorname{Im}\left(\Sigma_{L}\right)=2 \Delta_{L}, \\
& \Gamma_{R}=-2 \operatorname{Im}\left(\Sigma_{R}\right)=2 \Delta_{R},
\end{aligned}
$$

where $G$ represents the retarded Green's function of the system with the leads included and $\Gamma_{L}$ and $\Gamma_{R}$ are the coupling matrices of the system to the left and right leads, respectively. Since the matrix $\Gamma_{L}$ is only nonzero in the upper left matrix block 11 and $\Gamma_{R}$ only in the lower right matrix block $N N$, we can write

$$
\mathcal{T}=\operatorname{Tr}(Q), \quad Q=\Gamma_{11} G_{1 N} \Gamma_{N N}\left(G_{1 N}\right)^{\dagger},
$$

with $\Gamma_{11}$ the matrix block of $\Gamma_{L}$ at position $11, \Gamma_{N N}$ the matrix block of $\Gamma_{R}$ at position $N N$, and $G_{k l}$ the matrix block $k l$ of the matrix $G$. Symmetry can be used to blockdiagonalize the Hamiltonian $\mathcal{H}+\Sigma$ of the system, where we assume that $\Sigma$ has at least the symmetry of $\mathcal{H}$. Then of course the matrices $G, \Gamma_{L}, \Gamma_{R}$ will be block-diagonalized and as a consequence also the matrices $G_{1 N}, \Gamma_{11}, \Gamma_{N N}$. So the matrix $Q$ will be block-diagonalized into block matrices $Q_{j}$. It is now obvious that

$$
\mathcal{T}=\sum_{j} \mathcal{T}_{j}
$$

with

$$
\mathcal{T}_{j}=\operatorname{Tr}\left(Q_{j}\right) .
$$

In other words, the transmission can be split into contributions of different symmetry. In order to single out the contribution of individual channels to the transmission one can further diagonalize the matrix $Q .{ }^{21,22}$

In general, the self-energy is complex, but if it is constant in energy like we assume, one can prove via the Kramers-Krönig transformation ${ }^{23}$ that $\Lambda=0-$ i.e., that $\Sigma$ is purely imaginary.

To calculate $G_{1 N}$ we use the following relationship (see Appendix A):

$$
G_{1 N}=\left[T_{11}\left(E+0^{+} i\right)\right]^{-1} .
$$

Note that according to this method it is not necessary to calculate the scattering matrix ${ }^{17}$ to obtain the transmission.

\section{APPLICATION TO NANOTUBES}

\section{A. General block structure}

An infinite $(n, m)$ nanotube is characterized by the circumferential vector $\mathbf{C}_{\mathrm{h}}=n \mathbf{a}_{1}+m \mathbf{a}_{2} ; \mathbf{a}_{1}$ and $\mathbf{a}_{2}$ are the unit vectors as defined in Fig. 1(a) where an example is shown for a $(4,2)$ tube. The order of the principal axis is equal to $\mathcal{G}$, the greatest common divisor of $n$ and $m$. We use a simple basis set of one $p_{z}$ orbital per carbon atom and a Hamiltonian that includes only nearest-neighbor interactions-i.e., a simple orthogonal tight-binding model. But we allow the introduction of curvature: for a chiral tube there are three different hopping parameters $\beta_{1}, \beta_{2}, \beta_{3}$ as indicated in Fig. 1(a), 


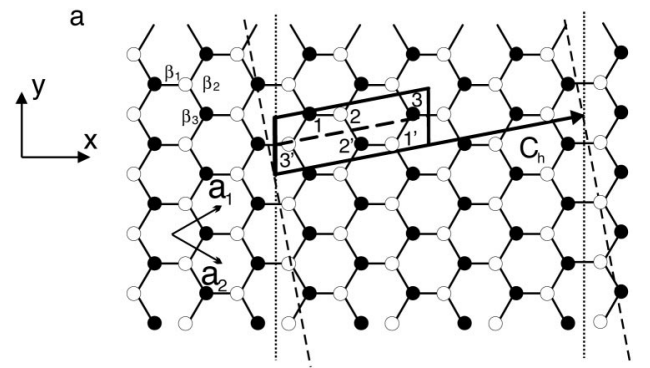

b

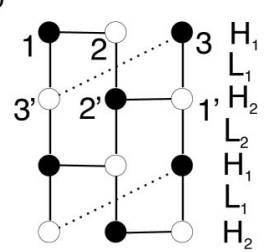

FIG. 1. (a) The vector $\mathbf{C}_{\mathrm{h}}=n a_{1}+m a_{2}$ defines the strip of an infinite nanotube, in this case for $n=4, m=2$. The nanotube can be obtained by cutting the strip defined by the dashed lines and gluing the ends or by first cutting the strip defined by the dotted lines. In the latter case, the parallelogram (thick line) defined by $a_{1}-a_{2}$ and $\mathbf{C}_{\mathrm{h}} / 2$ is a building block of this strip. The parallelogram has additional boundary conditions along $\mathbf{C}_{\mathrm{h}}$ with a phase factor $\exp (2 i \lambda)$ for the hopping parameter at the junction. The transfer matrix can be defined by appointing the atoms $(1,2,3)$ in the upper half of the unit parallelogram to $H_{1}$ and the atoms $\left(1^{\prime}, 2^{\prime}, 3^{\prime}\right)$ in the lower half to $H_{2}$. (b) Graphical scheme for defining the corresponding transfer matrix. The dotted diagonal line represents a hopping with phase $\exp (2 i \lambda)$ which makes the matrix $L_{1}$ complex.

while for an achiral tube (zigzag and armchair) two of them become equal due to its mirror symmetry. Normally the patch of the infinite tube on the graphite sheet is defined by two lines perpendicular to $\mathbf{C}_{h}$, one going through the origin and the other through the top of this vector. But when one defines a patch by two lines going through the same two points, but now in the direction $\mathbf{a}_{1}-\mathbf{a}_{2}$, exactly the same infinite tube is obtained. This patch can be tiled with parallelograms $\mathrm{P}_{u k}$ defined by the parallelogram $\mathrm{P}_{00}=\left\{\boldsymbol{0},\left(\mathbf{a}_{1}\right.\right.$ $\left.\left.-\mathbf{a}_{2}\right),(1 / \mathcal{G}) \mathbf{C}_{\mathrm{h}},(1 / \mathcal{G}) \mathbf{C}_{\mathrm{h}}+\left(\mathbf{a}_{1}-\mathbf{a}_{2}\right)\right\}$ and the displacement vector $u\left(\mathbf{a}_{1}-\mathbf{a}_{2}\right)+k(1 / \mathcal{G}) \mathbf{C}_{\mathrm{h}}$. Here $\mathcal{G}$ represents the greatest common divisor of $n$ and $m$. We represent an atomic orbital by $\chi_{r k j}$, where the labeling is defined by the principal axis $\mathrm{C}_{\mathcal{G}}$, the translation $\mathrm{T}_{\mathbf{a}_{1}-\mathbf{a}_{2}}$, and a $\mathrm{C}_{2}$ which is defined in the middle of each $\mathrm{P}_{u k}$ :

$$
\begin{aligned}
& \hat{\mathrm{C}}_{\mathcal{G}} \chi_{r k j}=\chi_{r k+1 j}, \\
& \hat{\mathrm{T}}_{\mathbf{a}_{1}-\mathbf{a}_{2}} \chi_{r k j}=\chi_{r k j+2}, \\
& \hat{\mathrm{C}}_{2} \chi_{r k 2 u-1}=\chi_{r k 2 u} .
\end{aligned}
$$

So $\mathrm{C}_{\mathcal{G}}$ relates two atoms of parallelograms $\mathrm{P}_{u k}$ and $\mathrm{P}_{u k+1}$ and $\mathrm{T}_{\mathbf{a}_{1}-\mathbf{a}_{2}}$ two atoms of parallelograms $\mathrm{P}_{u k}$ and $\mathrm{P}_{u+1 k}$, while $\mathrm{C}_{2}$ relates an atom in the lower half of the parallelogram with one in the upper half. The index $r$ counts the orbitals in one half of the parallelogram and runs from 1 to $(n+m) / \mathcal{G}$. The index $k$ runs from 1 to $\mathcal{G}$. First, rotational symmetry is used to block-diagonalize the Hamiltonian $\mathcal{H}$ of the nanotube to $\mathcal{G}$ block matrices $\mathcal{H}(\lambda)$. This is identical with taking the following symmetry adapted basis orbitals:

$$
\begin{aligned}
& \Phi_{r j}(\lambda)=\frac{1}{\sqrt{\mathcal{G}}} \sum_{k=0}^{\mathcal{G}-1} e^{-k 2 i \lambda} \chi_{r k j}, \\
& \lambda=\frac{\pi l}{\mathcal{G}}, \\
& \left\{\begin{array}{l}
l=-\frac{\mathcal{G}}{2}+1,-\frac{\mathcal{G}}{2}+2, \ldots, \frac{\mathcal{G}}{2}, \text { for } \mathcal{G} \text { even, } \\
l=-\frac{\mathcal{G}-1}{2},-\frac{\mathcal{G}-1}{2}+1, \ldots, \frac{\mathcal{G}-1}{2}, \text { for } \mathcal{G} \text { odd } .
\end{array}\right.
\end{aligned}
$$

Each $\mathcal{H}(\lambda)$ corresponds now to a "tube" defined by $(1 / \mathcal{G}) \mathbf{C}_{\mathrm{h}}$. This infinite tube can be thought of as a strip, obtained by tiling an infinite number of parallelograms $\mathrm{P}_{u 0}$ forming a ladder in the $y$ direction. The parallelogram has additional boundary conditions along $(1 / \mathcal{G}) \mathbf{C}_{\mathrm{h}}$ with a phase factor $\exp (2 i \lambda)$ for the hopping parameters at the junction. The matrix $\mathcal{H}(\lambda)$ is a tridiagonal block matrix with the following general repetitive form:

$$
\mathcal{H}(\lambda)=\left(\begin{array}{cccccc} 
& \ldots & & & & \\
\ldots & H_{1} & L_{1} & 0 & & \\
& L_{1}^{\dagger} & H_{2} & L_{2} & 0 & \\
& 0 & L_{2}^{\dagger} & H_{1} & L_{1} & \\
& & 0 & L_{1}^{\dagger} & H_{2} & \ldots \\
& & & & \ldots &
\end{array}\right) .
$$

In other words, each translational unit itself is composed of two internal dynamics matrices $H_{1}$ and $H_{2}$ interconnected by matrices $L_{1}$ and $L_{2}$. The choice of these matrices is not unique. Considering our basic parallelogram $\mathrm{P}_{00}$ with each corner in the middle of an hexagon, one can, for example, appoint all the atoms above the line $\left\{\frac{1}{2}\left(\mathbf{a}_{1}-\mathbf{a}_{2}\right),(1 / \mathcal{G}) \mathbf{C}_{\mathrm{h}}\right.$ $\left.+\frac{1}{2}\left(\mathbf{a}_{1}-\mathbf{a}_{2}\right)\right\}$ to $H_{1}$ and all atoms under to $H_{2}$. The size of the matrices $H_{1}, H_{2}, L_{1}, L_{2}$ is $(n+m) / \mathcal{G} \times(n+m) / \mathcal{G}$. The necessary condition $\operatorname{det}\left(L_{1,2}\right) \neq 0$ is also obeyed by this construction. A graphlike representation of $\mathcal{H}(\lambda)$ is shown in Fig. 1 for a $(2 n, n)$ tube. The corresponding one-step transfer matrices are

$$
\begin{aligned}
& T_{1}=\left(\begin{array}{cc}
L_{1}^{-1}\left(E I-H_{1}\right) & -L_{1}^{-1} L_{2}^{\dagger} \\
I & 0
\end{array}\right), \\
& T_{2}=\left(\begin{array}{cc}
L_{2}^{-1}\left(E I-H_{2}\right) & -L_{2}^{-1} L_{1}^{\dagger} \\
I & 0
\end{array}\right) .
\end{aligned}
$$

Now we consider instead of an infinite tube a finite tube:

$$
\mathcal{H}(\lambda)=\left(\begin{array}{cccccc}
H_{1} & L_{1} & 0 & & & \\
L_{1}^{\dagger} & H_{2} & L_{2} & & & \\
0 & L_{2}^{\dagger} & H_{1} & \ldots & & \\
& & \ldots & & & 0 \\
& & & \ldots & H_{v} & L_{v} \\
& & & 0 & L_{v}^{\dagger} & H_{w}
\end{array}\right) .
$$




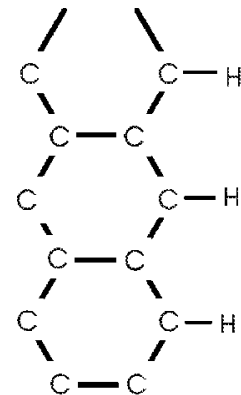

(a)

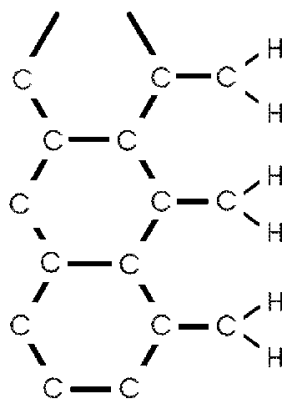

(b)
FIG. 2. (a) Hydrogen end of a zigzag tube. (b) Methylene end of a zigzag tube.

The corresponding transfer matrix has the following general form for an isolated tube for the two kinds of terminations:

$$
\begin{aligned}
& T=\left(\begin{array}{cc}
L_{1} & 0 \\
0 & I
\end{array}\right) T_{1}\left(T_{2} T_{1}\right)^{p}\left(\begin{array}{cc}
I & 0 \\
0 & \left(L_{2}^{\dagger}\right)^{-1}
\end{array}\right), \quad w=1, v=2, \\
& T=\left(\begin{array}{cc}
L_{2} & 0 \\
0 & I
\end{array}\right)\left(T_{2} T_{1}\right)^{p}\left(\begin{array}{cc}
I & 0 \\
0 & \left(L_{2}^{\dagger}\right)^{-1}
\end{array}\right), \quad w=2, v=1 .
\end{aligned}
$$

The start and end matrices in Eqs. (20) are necessary because in the matrix $\mathcal{H}(\lambda)$ we set $L_{0}=L_{N}=I .{ }^{17}$ The coefficient vectors $\mathbf{c}_{j}$ of a wave function are defined as

$$
\mathbf{c}_{j}=\left(\begin{array}{c}
c_{1 j} \\
\vdots \\
c_{[(n+m) / \mathcal{G}] j}
\end{array}\right),
$$

where the coefficients $c_{r j}$ are defined for a wave function expressed as a linear combination of the symmetry adapted basis orbitals:

$$
\psi(\lambda)=\sum_{j=1}^{N} \sum_{r=1}^{(n+m) / \mathcal{G}} c_{r j} \Phi_{r j}(\lambda),
$$

where $N$ is twice the number of parallelograms $\mathrm{P}_{u 0}$ one encounters by moving from the beginning to the end of the tube in the direction $\mathbf{a}_{1}-\mathbf{a}_{2}$. When $N$ is even, the two ends of the tube are identical. For a tube connected to two leads via the edges one has to include the self-energy of the left lead and of the right lead. If we suppose that it has the same rotational symmetry as $\mathcal{H}$ we can block-diagonalize it:

$$
\begin{aligned}
& \left(\Sigma_{11}(\lambda)\right)_{r r^{\prime}}=\frac{1}{\mathcal{G}} \sum_{k=0}^{\mathcal{G}-1} e^{2 i \lambda k}\left\langle\chi_{r 01}\left|\Sigma_{L}\right| \chi_{r^{\prime} k 1}\right\rangle, \\
& \left(\Sigma_{N N}(\lambda)\right)_{r r^{\prime}}=\frac{1}{\mathcal{G}} \sum_{k=0}^{\mathcal{G}-1} e^{2 i \lambda k}\left\langle\chi_{r 0 N}\left|\Sigma_{L}\right| \chi_{r^{\prime} k N}\right\rangle .
\end{aligned}
$$

The matrices $\Sigma_{L}$ and $\Sigma_{R}$ are of the same size as $\mathcal{H}$-i.e., $N(n+m) \times N(n+m)$ - but they are nonzero only at the positions corresponding to the $(n+m) \times(n+m)$ matrix blocks $j=1$ and $j=N$, respectively. The matrices $\Sigma_{11}(\lambda)$ and $\Sigma_{N N}(\lambda)$ are of size $(n+m) / \mathcal{G} \times(n+m) / \mathcal{G}$ and are added to the first and last matrix blocks of $\mathcal{H}(\lambda)$. The transfer matrix becomes (we have omitted the dependence on $\lambda$ for simplicity)

$$
\begin{aligned}
T= & {\left[\left(\begin{array}{cc}
L_{w} & 0 \\
0 & I
\end{array}\right) T_{w}-\left(\begin{array}{cc}
\Sigma_{N N} & 0 \\
0 & 0
\end{array}\right)\right] \cdots T_{2} T_{1} T_{2} } \\
& \times\left[T_{1}\left(\begin{array}{cc}
I & 0 \\
0 & \left(L_{2}^{\dagger}\right)^{-1}
\end{array}\right)-\left(\begin{array}{cc}
L_{1}^{-1} \Sigma_{11} & 0 \\
0 & 0
\end{array}\right)\right],
\end{aligned}
$$

which gives, for the element $T_{11}$,

$$
\begin{aligned}
T_{11}= & L_{1}\left\{T_{1}\left(T_{2} T_{1}\right)^{p}\right\}_{11}-\Sigma_{N N}\left\{\left(T_{2} T_{1}\right)^{p}\right\}_{11} \\
& -L_{1}\left\{\left(T_{1} T_{2}\right)^{p}\right\}_{11} L_{1}^{-1} \Sigma_{11} \\
& +\Sigma_{N N}\left\{T_{2}\left(T_{1} T_{2}\right)^{p-1}\right\}_{11} L_{1}^{-1} \Sigma_{11}, \\
T_{11}= & L_{2}\left\{\left(T_{2} T_{1}\right)^{p}\right\}_{11}-\Sigma_{N N}\left\{\left(T_{1} T_{2}\right)^{p-1} T_{1}\right\}_{11} \\
& -L_{2}\left\{T_{2}\left(T_{1} T_{2}\right)^{p-1}\right\}_{11} L_{1}^{-1} \Sigma_{11} \\
& +\Sigma_{N N}\left\{\left(T_{1} T_{2}\right)^{p-1}\right\}_{11} L_{1}^{-1} \Sigma_{11},
\end{aligned}
$$

for $w=1$ and 2, respectively. It is useful to diagonalize $T_{2} T_{1}$ and $T_{1} T_{2}$ first:

$$
\begin{aligned}
& T_{2} T_{1}=S T_{D} S^{-1} \Rightarrow\left(T_{2} T_{1}\right)^{r}=S T_{D}^{r} S^{-1}, \\
& T_{1} T_{2}=S^{\prime} T_{D} S^{\prime-1} \Rightarrow\left(T_{1} T_{2}\right)^{r}=S^{\prime} T_{D}^{r} S^{\prime-1},
\end{aligned}
$$

with $T_{D}$ a diagonal matrix with the eigenvalues of $T_{2} T_{1}$ on its diagonal. This general scheme is applied to obtain $T_{11}$ for zigzag and armchair tubes, isolated or attached to the leads.

\section{B. Zigzag tubes}

We consider two different types of termination of the $(n, 0)$ zigzag tube: (i) a carbon atom connected to two other carbons and one hydrogen $(\mathrm{H})$ and (ii) a carbon atom connected to one carbon and two hydrogens-i.e., a methylene group (M) as represented in Fig. 2. This makes three kinds of tubes possible: hydrogen-hydrogen ended ( $\mathrm{HH})$, methylene-methylene ended (MM), and hydrogenmethylene ended (HM). To characterize our nanotube we use the notation

$$
(n, N, \mathrm{XY}) \text {, }
$$

where $n$ is the order of the principal rotational axis, since $n=\mathcal{G}$. Since one-half of a parallelogram $\mathrm{P}_{u k}$ contains only one atom, the total number of atoms of the tube is equal to $n N$. The symbols $\mathrm{X}, \mathrm{Y}$ can be $\mathrm{H}$ or $\mathrm{M}$ where $\mathrm{X}$ is used for the first atom and $\mathrm{Y}$ for the last. Note that $N$ is even for $\mathrm{HH}$ or MM tubes and odd for HM tubes. Furthermore, HM tubes have only $\mathrm{C}_{n v}$ symmetry while $\mathrm{HH}$ and $\mathrm{MM}$ tubes have $\mathrm{D}_{n d}$ symmetry if $N$ is divisible by 4 and $\mathrm{D}_{n h}$ symmetry otherwise. The concept of the HM tubes was also used as a unit for junctions of ribbons, where they are called bearded ribbons. ${ }^{24}$ We can use the rotational symmetry to blockdiagonalize the tight-binding Hamiltonian of the zigzag tube, $\mathcal{H}_{\mathrm{Z}}$, to $n$ submatrices $\mathcal{H}_{\mathrm{Z}}(\lambda)$, with

$\lambda=\frac{\pi l}{n},\left\{\begin{array}{l}l=-\frac{n}{2}+1,-\frac{n}{2}+2, \ldots, \frac{n}{2}, \text { for } n \text { even, } \\ l=-\frac{n-1}{2},-\frac{n-1}{2}+1, \ldots, \frac{n-1}{2}, \text { for } n \text { odd. }\end{array}\right.$ 

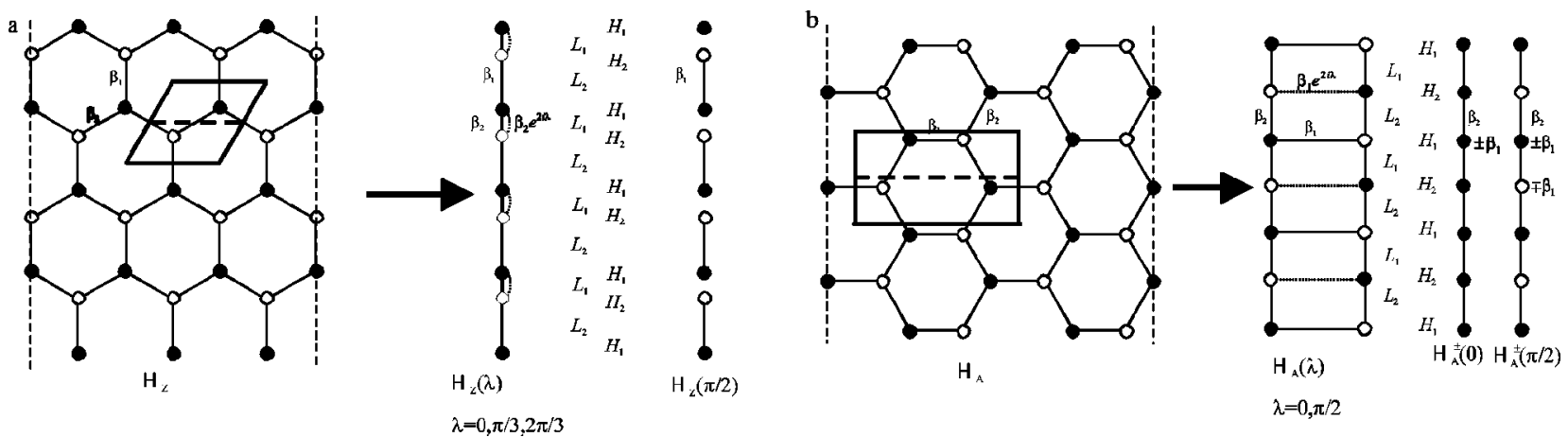

FIG. 3. (a) (3,9,HM) zigzag tube, its conversion to the graphical representation of $\mathcal{H}_{\mathrm{Z}}(\lambda)$, and the assignment to the matrices $H_{1}, H_{2}$ and $L_{1}, L_{2}$. The special example $\mathcal{H}_{\mathrm{Z}}(\pi / 2)$ is also given. (b) $(2,7)$ armchair tube, its conversion to the graphical representation of $\mathcal{H}_{\mathrm{A}}(\lambda), \mathcal{H}_{\mathrm{A}}^{ \pm}(0)$, and $\mathcal{H}_{\mathrm{A}}^{ \pm}(\pi / 2)$ and the assignment to the matrices $H_{1}, H_{2}$ and $L_{1}, L_{2}$. The dotted lines in the graphs indicate a hopping with phase $\exp (2 i \lambda)$.

A graphical representation of the conversion $\mathcal{H}_{\mathrm{Z}} \rightarrow \mathcal{H}_{\mathrm{Z}}(\lambda)$ in the case of a HM tube is given in Fig. 3(a). The corresponding transfer matrix is a $2 \times 2$ matrix. Application of Eq. (19) to a zigzag tube that begins with a hydrogen side leads to

$$
\begin{aligned}
& L_{1}=\beta_{2}+\beta_{2} e^{2 i \lambda}=2 \beta_{2} \cos \lambda e^{i \lambda}, \\
& L_{2}=\beta_{1}, \\
& H_{1}=H_{2}=0 .
\end{aligned}
$$

We have set the energy of the carbon atoms equal to zero. For a tube that begins with a methylene end one has $L_{1}$ $=\beta_{1}$ and $L_{2}=2 \beta_{2} \cos \lambda e^{i \lambda}$. However, we can get rid of the phase factor by the following phase transformation of the symmetry-adapted basis orbitals:

atom 1 at hydrogen side: $\left\{\widetilde{\Phi}_{2 u}(\lambda), \widetilde{\Phi}_{2 u+1}(\lambda)\right\}$

$$
=e^{-u i \lambda}\left\{\Phi_{2 u}(\lambda), \Phi_{2 u+1}(\lambda)\right\},
$$

atom 1 at methylene side: $\left\{\widetilde{\Phi}_{2 u+1}(\lambda), \widetilde{\Phi}_{2 u+2}(\lambda)\right\}$

$$
=e^{u i \lambda}\left\{\Phi_{2 u+1}(\lambda), \Phi_{2 u+2}(\lambda)\right\} .
$$

The new off-diagonal elements are $b_{1}=2 \beta_{2} \cos \lambda$ and $b_{2}$ $=\beta_{1}$ for a tube that begins with a hydrogen end and vice versa for a tube beginning with a methylene end. Using these basis orbitals, it follows that the repeating unit is described by

$$
T_{2} T_{1}=\frac{1}{b_{1}}\left(\begin{array}{cc}
\frac{1}{b_{2}}\left(E^{2}-b_{1}^{2}\right) & -E \\
E & -b_{2}
\end{array}\right) .
$$

Under the substitution

$$
\begin{aligned}
E & =-\sigma_{\mu} \sqrt{4 \beta_{1} \beta_{2} \cos \lambda \cos \varphi+\beta_{1}^{2}+4 \beta_{2}^{2} \cos ^{2} \lambda} \\
& =-\sigma_{\mu} \sqrt{2 b_{1} b_{2} \cos \varphi+b_{1}^{2}+2 b_{2}^{2}},
\end{aligned}
$$

with $\sigma_{\mu}=1$ for bonding states and -1 for antibonding states, the diagonal matrix $T_{D}$ is expressed as

$$
T_{D}=\left(\begin{array}{cc}
e^{i \varphi} & 0 \\
0 & e^{-i \varphi}
\end{array}\right),
$$

with

$$
\cos \varphi=\frac{E^{2}-\beta_{1}^{2}-4 \beta_{2}^{2} \cos ^{2} \lambda}{4 \beta_{1} \beta_{2} \cos \lambda}
$$

and via construction of the diagonalizing matrix $S$ in Eq. (26), ${ }^{17}$ we find, for $\left(T_{2} T_{1}\right)^{r}$,

$$
\left(T_{2} T_{1}\right)^{r}=\left(\begin{array}{l}
\frac{\sin (r+1) \varphi}{\sin \varphi}+\frac{b_{2}}{b_{1}} \frac{\sin r \varphi}{\sin \varphi}-\frac{E}{b_{1}} \frac{\sin r \varphi}{\sin \varphi} \\
\frac{E}{b_{1}} \frac{\sin r \varphi}{\sin \varphi}-\frac{\sin (r-1) \varphi}{\sin \varphi}-\frac{b_{2}}{b_{1}} \frac{\sin r \varphi}{\sin \varphi}
\end{array}\right)
$$

and similar expressions for $\left(T_{1} T_{2}\right)^{r}, T_{1}\left(T_{2} T_{1}\right)^{r}$, and $\left(T_{2} T_{1}\right)^{r} T_{2}$.

For isolated zigzag tubes the determinants of $E$ $-\mathcal{H}_{\mathrm{Z}}(\lambda)$ are found by combining Eqs. (4) and (20) and the expression for $\left(T_{2} T_{1}\right)^{N / 2}$ in the case of HH and MM tubes or $T_{1}\left(T_{2} T_{1}\right)^{(N-1) / 2}$ in the case of HM tubes:

$$
D_{\mathrm{Z}}^{\mathrm{XX}}(\lambda, N)=\left(b_{1} b_{2}\right)^{N / 2} \frac{\sin \frac{N+2}{2} \varphi+\frac{b_{2}}{b_{1}} \sin \frac{N}{2} \varphi}{\sin \varphi},
$$

$\mathrm{XX}=\mathrm{HH}, \mathrm{MM}$,

$$
D_{\mathrm{Z}}^{\mathrm{HM}}(\lambda, N)=E\left(b_{1} b_{2}\right)^{(N-1) / 2} \frac{\sin \frac{N+1}{2} \varphi}{\sin \varphi} .
$$

Eigenenergies are the roots of the $D_{\mathrm{Z}}^{\mathrm{XY}}=0$ equation. The coefficients $c_{j}^{\mathrm{XY}}$ of the (unnormalized) wave function of $\mathcal{H}_{\mathrm{Z}}(\lambda)$ can be written as

$\left\{c_{2 u}^{\mathrm{XY}}, c_{2 u+1}^{\mathrm{XY}}\right\}=\frac{c_{1}^{\mathrm{XY}}}{\sin \varphi}\left\{\frac{-E}{b_{1}} \sin u \varphi, \sin (u+1) \varphi+\frac{b_{2}}{b_{1}} \sin u \varphi\right\}$,

with $c_{j}^{\mathrm{HY}}$ the $j$ th coefficient for a system where atom 1 is on a hydrogen end, $c_{j}^{\mathrm{MY}}$ for a system where atom 1 is on a methylene end. By not specifying $c_{1}^{\mathrm{XY}}$ there is one degree of freedom left for normalization. Note that, since there is only one atom in one-half of the parallelogram $\mathrm{P}_{u 0}$, we have omitted the index $r ; u$ counts couples of coefficients and runs from 0 to $(N-1) / 2$ for $\mathrm{HM}$ tubes and from 0 to $N / 2$ for 
$\mathrm{HH}$ and $\mathrm{MM}$ tubes. It is readily seen that $c_{0}^{\mathrm{XY}}=0$, like it should. We have also the condition $c_{N+1}^{\mathrm{XY}}=0$.

For HM tubes, one can determine the solutions of $D_{\mathrm{Z}}^{\mathrm{HM}}(\lambda, N)=0$ exactly: hence in this case the transfer matrix method leads directly to the solution of the eigenvalue problem:

$$
\begin{aligned}
& \frac{\sin \frac{N+1}{2} \varphi}{\sin \varphi}=0 \Leftrightarrow \varphi=f \frac{2 \pi}{N+1}, \quad f=1, \ldots, \frac{N-1}{2}, \\
& E=0 \Leftrightarrow \varphi=\pi-i \alpha,
\end{aligned}
$$

with

$$
\cosh \alpha=\frac{1}{2}\left(\frac{\beta_{1}}{2 \beta_{2} \cos \lambda}+\frac{2 \beta_{2} \cos \lambda}{\beta_{1}}\right) .
$$

The first $(N-1) / 2$ solutions correspond to nondecaying wave functions, since $\varphi$ is real. Note that these solutions are also contained in the spectrum of a polyhex carbon torus with indices $(n, 0,(N+1) / 2,-N+1)$ following the convention developed by Ceulemans et al. ${ }^{25}$ But the last solution at energy zero corresponds in general to a decaying, nonbonding wave function, since $\frac{1}{2}\left(\beta_{1} / 2 \beta_{2} \cos \lambda+2 \beta_{2} \cos \lambda / \beta_{1}\right) \geqslant 1$, $\alpha \geqslant 0$. Counting from the hydrogen end, the coefficients of the normalized wave functions of $\mathcal{H}_{\mathrm{Z}}^{\mathrm{HM}}(\lambda)$ have the following form:

$$
\begin{aligned}
\left\{c_{2 u}^{\mathrm{HM}}, c_{2 u+1}^{\mathrm{HM}}\right\}= & \sqrt{\frac{2}{N+1}}\{\sin (u \varphi), \\
& \left.\frac{2 \beta_{2} \cos \lambda \sin (u+1) \varphi+\beta_{1} \sin (u \varphi)}{-E_{\mu \varphi}}\right\}
\end{aligned}
$$

for the nondecaying wave functions and

$$
\begin{aligned}
\left\{c_{2 u}^{\mathrm{HM}}, c_{2 u+1}^{\mathrm{HM}}\right\}= & \sqrt{\frac{\left(\frac{2 \beta_{2} \cos \lambda}{\beta_{1}}\right)^{2}-1}{\left(\frac{2 \beta_{2} \cos \lambda}{\beta_{1}}\right)^{N+1}-1}} \\
& \times\left(-\frac{2 \beta_{2} \cos \lambda}{\beta_{1}}\right)^{u}\{0,1\}
\end{aligned}
$$

for the decaying ones. For $\lambda=0$ this wave function decreases from the methylene end towards the hydrogen end. When $\lambda$ grows, the wave function gradually penetrates more towards the bulk of the tube until at $\beta_{1} / 2 \beta_{2} \cos \lambda=1$ the wave function is totally extended. For still larger $\lambda$ the wave function decreases from the hydrogen end towards the methylene end. For $\lambda=\pi / 2, \quad\left(2 \beta_{2} \cos \lambda / \beta_{1}\right)^{u}=0$ except for $u=0$ and the wave function is completely localized on atom 1 of the hydrogen end. Note that for $N \rightarrow \infty, n \rightarrow \infty$, and $\beta_{1}=\beta_{2}$ one obtains the solutions of semi-infinite graphene with a hydrogen edge, including the edge states for $\pi / 2 \geqslant \lambda \geqslant \pi / 3 .{ }^{8}$ The introduction of curvature is important because, whereas in the symmetric case $\left(\beta_{1}=\beta_{2}\right)$ the nondecaying wave function of the finite ribbon or tube corresponding to $\lambda=\pi / 3$ was identified with the wave function at the Fermi level of graphene, ${ }^{8}$ this no longer holds: the wave function becomes a decaying one with exponent $\alpha=\operatorname{arccosh}\left[\frac{1}{2}\left(\beta_{1} / \beta_{2}\right.\right.$ $\left.\left.+\beta_{2} / \beta_{1}\right)\right]$. For the whole tube, there are $n$ decaying wave functions, one for every $\lambda$, all with energy zero.

The situation is different for the edge states of MM and $\mathrm{HH}$ tubes. We can write as two starting wave functions for $\mathcal{H}_{\mathrm{Z}}^{\mathrm{HH}}(\lambda)\left[\mathcal{H}_{\mathrm{Z}}^{\mathrm{MM}}(\lambda)\right]$ two edge states of type HM, one starting at the first hydrogen [methylene] edge, and the other starting at the other edge. This is of course only possible for $2 \beta_{2} \cos \lambda<\beta_{1}$ for HH or $2 \beta_{2} \cos \lambda>\beta_{1}$ for MM. The two wave functions, $\Psi_{\bullet}^{\mathrm{XY}}$ and $\Psi_{\mathrm{O}}^{\mathrm{YX}}, \mathrm{X} \neq \mathrm{Y}$, are orthogonal since they are nonzero only on black and white atoms, respectively. Correctly normalized and with the coefficients counted from the same end, they have the following form:

$$
\begin{aligned}
& \Psi_{\bullet}^{\mathrm{XY}}=\sqrt{\frac{a^{2}-1}{|a|^{N}-1} \sum_{u=0}^{N / 2-1} a^{u} \widetilde{\Phi}_{2 u+1}(\lambda),} \\
& \Psi_{\bigcirc}^{\mathrm{YX}}=\sqrt{\frac{a^{2}-1}{|a|^{N}-1} \sum_{u=1}^{N / 2} a^{N / 2-u} \widetilde{\Phi}_{2 u}(\lambda),}
\end{aligned}
$$

with $a=-b_{2} / b_{1}$. Calculating the interaction matrix is fairly easily when one realizes that the Hamiltonian can be splitted into two parts:

$$
\begin{aligned}
& \mathcal{H}_{\mathrm{Z}}^{\mathrm{XX}}=\mathcal{H}_{\mathrm{Z}}^{\mathrm{XY}}+\mathcal{H}_{\mathrm{Z}}^{\prime}, \\
& \mathcal{H}_{\mathrm{Z}}^{\prime}=b_{2}\left|\Phi_{N-1}\right\rangle\left\langle\Phi_{N}\left|+b_{2}\right| \Phi_{N}\right\rangle\left\langle\Phi_{N-1}\right|,
\end{aligned}
$$

and since $\Psi_{\bullet}^{\mathrm{HM}}$ is an eigenfunction of $\mathcal{H}_{\mathrm{Z}}^{\mathrm{HM}}$ with eigenvalue zero we have

$$
\begin{aligned}
\mathcal{H}_{\mathrm{Z}}^{\mathrm{XX}} \Psi_{\bullet}^{\mathrm{HM}}= & \mathcal{H}_{\mathrm{Z}}^{\prime} \Psi_{\bullet}^{\mathrm{XY}} \\
= & \left(b_{2}\left|\widetilde{\Phi}_{N-1}\right\rangle\left\langle\widetilde{\Phi}_{N}\left|+b_{2}\right| \widetilde{\Phi}_{N}\right\rangle\right. \\
& \left.\times\left\langle\widetilde{\Phi}_{N-1}\right|\right) \sqrt{\frac{a^{2}-1}{|a|^{N}-1} \sum_{u=0}^{N / 2-1} a^{u}\left|\Phi_{2 u+1}\right\rangle} \\
= & \sqrt{\frac{a^{2}-1}{|a|^{N}-1}} b_{2} a^{N / 2-1}\left|\widetilde{\Phi}_{N}\right\rangle
\end{aligned}
$$

so we get, for the matrix elements,

$$
\begin{aligned}
\left\langle\Psi_{\bullet}^{\mathrm{XY}}\left|\mathcal{H}_{\mathrm{Z}}^{\mathrm{XX}}\right| \Psi_{\bullet}^{\mathrm{XY}}\right\rangle & =0=\left\langle\Psi_{\bigcirc}^{\mathrm{YX}}\left|\mathcal{H}_{\mathrm{Z}}^{\mathrm{XX}}\right| \Psi_{\bigcirc}^{\mathrm{YX}}\right\rangle, \\
\left\langle\Psi_{\mathrm{O}}^{\mathrm{YX}}\left|\mathcal{H}_{\mathrm{Z}}^{\mathrm{XX}}\right| \Psi_{\bullet}^{\mathrm{XY}}\right\rangle & =\frac{a^{2}-1}{|a|^{N}-1} a^{N / 2-1} b_{2} \\
& =\left\langle\Psi_{\bullet}^{\mathrm{XY}}\left|\mathcal{H}_{\mathrm{Z}}^{\mathrm{XX}}\right| \Psi_{\bigcirc}^{\mathrm{YX}}\right\rangle .
\end{aligned}
$$

The interaction matrix has the form

$$
\left(\begin{array}{cc}
0 & \frac{|a|^{2}-1}{|a|^{N}-1} a^{N / 2-1} b_{2} \\
\frac{|a|^{2}-1}{|a|^{N}-1} a^{N / 2-1} b_{2} & 0
\end{array}\right)\left(\begin{array}{l}
d_{\bullet} \\
d_{\odot}
\end{array}\right)=E\left(\begin{array}{l}
d_{\bullet} \\
d_{\odot}
\end{array}\right),
$$

from which we find the energy spectrum and the eigenvectors:

$$
\begin{aligned}
& E= \pm \frac{a^{2}-1}{|a|^{N}-1}|a|^{N / 2-1}\left|b_{2}\right|, \\
& d_{\bullet}= \pm \operatorname{sgn}\left[b_{2} a^{N / 2-1}\right] d_{\bigcirc} .
\end{aligned}
$$



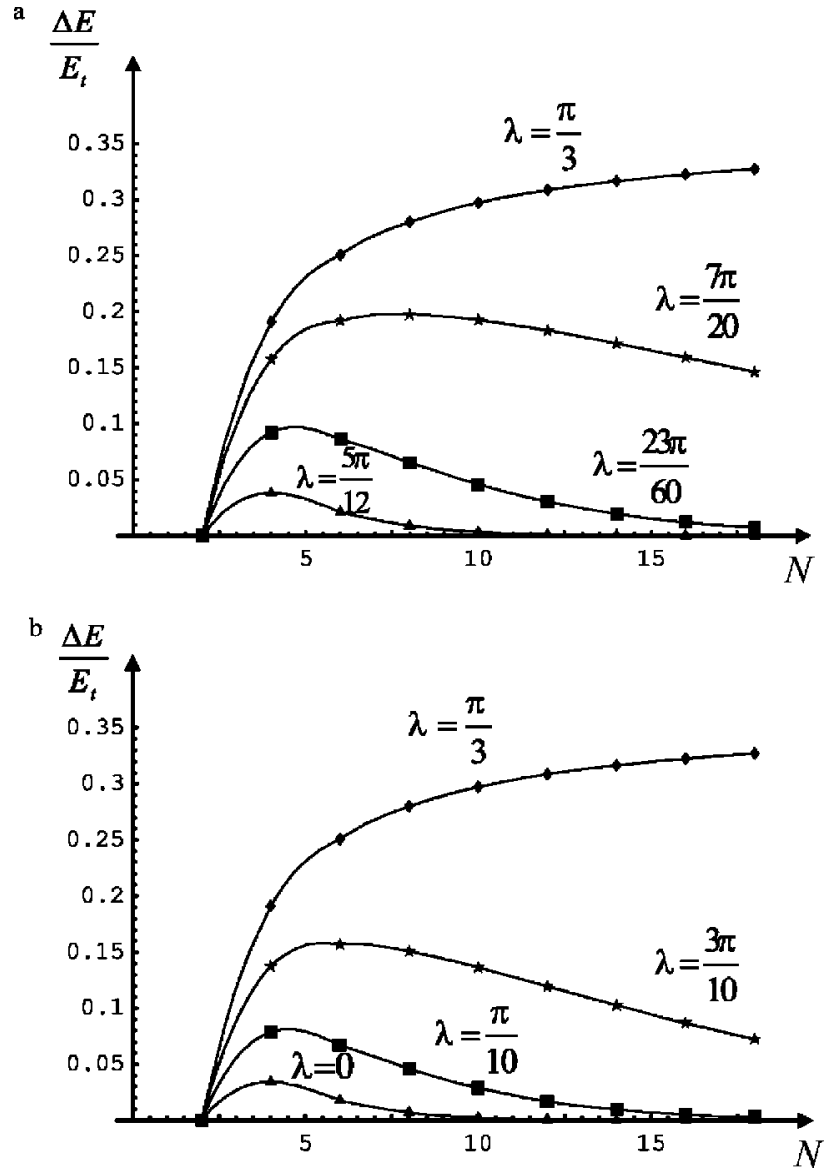

FIG. 4. Relative error of Eqs. (44) as a function of tube length. The effect of curvature is not included. (a) HH tube. (b) MM tube.

This procedure was also followed in Ref. 9 for $\mathrm{HH}$ ribbons without curvature, but incorrectly because the normalization and the phase shift of the wave function were not taken into account. So, for the MM tubes, two decaying wave functions exist for each $\lambda$ obeying the condition $\beta_{1} / 2 \beta_{2} \cos \lambda<1$, while for $\mathrm{HH}$ tubes two decaying wave functions exist for each $\lambda$ obeying the condition $\beta_{1} / 2 \beta_{2} \cos \lambda>1$, and their energy is small but nonzero except for HH tubes when $\lambda=\pi / 2$. In Fig. 4 we show the relative error of Eqs. (44) by plotting $\Delta E / E_{t}$ versus length for the case of no curvature, where $\Delta E$ is defined as

$$
\Delta E=E_{t}-E_{a},
$$

with $E_{t}$ the true value-i.e., the lowest positive eigenvalue obtained by solving the tight-binding matrix directly-and $E_{a}$ the approximate energy with positive sign obtained from Eqs. (44). It is not difficult to prove that, for $N=2, E_{a}=E_{t}$ and, for $\lambda=\pi / 2, E_{a}=E_{t}=0$. From Fig. 4 one can conclude that $E_{a}$ always underestimates the true value, that the approximation is worse when $|a|$ is closer to unity, and that for $|a| \neq 1$, the approximation converges to the true solution in the limit of large $N$.

Taking the limit $|a| \rightarrow 1$ one finds that $E_{a} \rightarrow(2 / N)|\beta|$, so there is a linear instead of an exponential decrease. The exact solution in this case reads

$$
E_{t}=2|\beta| \cos \left(\frac{N}{1+N} \frac{\pi}{2}\right) \approx \frac{\pi}{N}|\beta|
$$

[vide infra Eq. (48)], so the relative error $\Delta E / E_{t}$ goes asymptotically towards $(\pi-2) / \pi \approx 0.363$ for large lengths which can also be seen in Fig. 4. The absolute error is maximal for $|a|=1$ and $N=4$, and is equal to $\Delta E \approx 0.118 \beta$.

The band gap of $(2 q, N, \mathrm{HH})$ tubes due to these edge states is zero, while for the $(n, N, \mathrm{MM})$ tubes it is equal to

$$
2 \frac{\left(\frac{\beta_{1}}{2 \beta_{2}}\right)^{2}-1}{\left(\frac{\beta_{1}}{2 \beta_{2}}\right)^{N}-1}\left(\frac{\beta_{1}}{2 \beta_{2}}\right)^{N / 2-1}\left|\beta_{1}\right| \approx \frac{3}{2^{N / 2}}|\beta|
$$

in the limit of large $N$ and no curvature. The same result was obtained by Klein ${ }^{6}$ using an ansatz.

Infinite $(3 q, 0)$ zigzag tubes are metallic if one ignores curvature. The matrix block $\mathcal{H}_{\mathrm{Z}}(\pi / 3)$ is responsible for this metallicity. For finite tubes and symmetric hopping parameters, the equations $D^{\mathrm{HH}}(\pi / 3, N)=0$ and $D^{\mathrm{MM}}(\pi / 3, N)=0$ are analytically solvable for $\mathrm{HH}$ and $\mathrm{MM}$ tubes also:

$$
\begin{aligned}
D_{\mathrm{Z}}^{\mathrm{HH}, \mathrm{MM}}\left(\frac{\pi}{3}, N\right) & =\beta^{N} \frac{\sin \frac{N+2}{2} \varphi+\sin \frac{N}{2} \varphi}{\sin \varphi} \\
& =0 \Rightarrow \varphi=f \frac{2 \pi}{1+N}, \quad f=1, \ldots, \frac{N}{2},
\end{aligned}
$$

and via Eq. (32) one obtains

$$
E=\sigma_{\mu} 2|\beta| \cos \left(\frac{f \pi}{1+N}\right) .
$$

So the band gap for nondecaying wave functions of both $\mathrm{HH}$ and $\mathrm{MM}$ tubes is equal to

$$
4|\beta| \cos \left(\frac{N}{1+N} \frac{\pi}{2}\right) \approx \frac{2|\beta| \pi}{N}, \quad \text { when } N \rightarrow \infty \text {. }
$$

These results for $\mathrm{MM}$ and $\mathrm{HH}$ were already obtained by Klein ${ }^{6}$ and Wakabayashi et al., ${ }^{9}$ respectively. For the band gap of HM tubes, on the other hand, we have

$$
4|\beta| \cos \left(\frac{N-1}{N+1} \frac{\pi}{2}\right) \approx \frac{4|\beta| \pi}{N}, \quad \text { when } N \rightarrow \infty .
$$

Thus, for large lengths, the band gap of the three kinds of tubes is inversely proportional to tube length but it is twice as large for HM tubes as for $\mathrm{HH}$ and MM tubes.

To conclude, we investigate the band structure for $\lambda=\pi / 2$ for the three tubes. The corresponding graph for a HM tube is shown in Fig. 3(a):

$$
\begin{aligned}
& D_{\mathrm{Z}}^{\mathrm{MM}}\left(\frac{\pi}{2}, N\right)=\left(E^{2}-\beta_{1}^{2}\right)^{N / 2}, \\
& D_{\mathrm{Z}}^{\mathrm{HH}}\left(\frac{\pi}{2}, N\right)=E^{2}\left(E^{2}-\beta_{1}^{2}\right)^{N / 2-1}, \\
& D_{\mathrm{Z}}^{\mathrm{HM}}\left(\frac{\pi}{2}, N\right)=E\left(E^{2}-\beta_{1}^{2}\right)^{(N-1) / 2} .
\end{aligned}
$$


These determinants can be found directly from $\mathcal{H}_{Z}(\pi / 2)$ or from Eqs. (35) by taking the limit $\lambda \rightarrow \pi / 2$. The eigenenergies of $\mathcal{H}_{\mathrm{Z}}(\pi / 2)$ are then readily obtained. Also the coefficients of the wave functions can be found from Eq. (36) by taking the limit $\lambda \rightarrow \pi / 2$. For the wave functions corresponding to $E= \pm \beta_{1}$ we have (in the case of HM tubes we count from the hydrogen end)

$$
\begin{aligned}
& \left\{c_{2 u}^{\mathrm{HH}}, c_{2 u+1}^{\mathrm{HH}}\right\}=\sqrt{\frac{2}{N}} \sin u \varphi\left\{1, \sigma_{\mu}\right\}, \\
& \varphi=f \frac{2 \pi}{N}, \quad f=1, \ldots, \frac{N}{2}-1, \\
& \left\{c_{2 u}^{\mathrm{MM}}, c_{2 u+1}^{\mathrm{MM}}\right\}=\sqrt{\frac{2}{N+2}}\left\{\sin u \varphi, \sigma_{\mu} \sin (u+1) \varphi\right\}, \\
& \varphi=f \frac{2 \pi}{N+2}, \quad f=1, \ldots, \frac{N}{2}, \\
& \left\{c_{2 u}^{\mathrm{HM}}, c_{2 u+1}^{\mathrm{HM}}\right\}=\sqrt{\frac{2}{N+1}} \sin u \varphi\left\{1, \sigma_{\mu}\right\}, \\
& \varphi=f \frac{2 \pi}{N+1}, \quad f=1, \ldots, \frac{N-1}{2} .
\end{aligned}
$$

For the decaying wave functions corresponding to $E=0$ we have

$$
\begin{aligned}
& c_{1}^{\mathrm{HH}}= \pm c_{N}^{\mathrm{HH}}= \pm \frac{1}{\sqrt{2}}, \quad c_{j}^{\mathrm{HH}}=0, \quad j=2, \ldots, N-1, \\
& c_{1}^{\mathrm{HM}}=1, \quad c_{j}^{\mathrm{HM}}=0, \quad j=2, \ldots, N .
\end{aligned}
$$

The high degeneracy at $\pm \beta_{1}$ is actually accidental, because we assume the same parameter $\beta_{1}$ for all the bonds parallel to the nanotube axis although they are not all symmetry related. When $E=0$, the wave functions of $\mathrm{HH}$ tubes are completely localized at the two edges of the nanotube, while for HM tubes they are completely localized on the $\mathrm{H}$ edge. The high number of states around $\pm \beta_{1}$ suggests that the transmission of the nanotubes is high around this energy and because of the states at $E=0$ one could expect that this will make a "metal" of the tube, but both assumptions are false, as we shall see below (Sec. IV).

\section{Armchair tubes}

Finite $(n, n)$ armchair tubes can be characterized by the order of the principal rotation axis $n$ and the number of rings $N$ :

$$
(n, N) \text {. }
$$

When $N$ is odd the symmetry of the tube is $D_{n h}$ : otherwise, it is $D_{n d}$. The matrix $\mathcal{H}_{\mathrm{A}}$ can again be block-diagonalized to $n$ submatrices $\mathcal{H}_{\mathrm{A}}(\lambda)$. A graphical representation of $\mathcal{H}_{\mathrm{A}}(\lambda)$ is given in Fig. 3(b). The corresponding transfer matrix is of size $4 \times 4$. Application of Eq. (19) to an armchair tube leads to

$$
\begin{aligned}
& L_{1}=L_{2}=\left(\begin{array}{cc}
0 & \beta_{2} \\
\beta_{2} & 0
\end{array}\right), \\
& H_{1}=\left(\begin{array}{cc}
0 & \beta_{1} \\
\beta_{1} & 0
\end{array}\right), \\
& H_{2}=\left(\begin{array}{cc}
0 & \beta_{1} e^{2 i \lambda} \\
\beta_{1} e^{-2 i \lambda} & 0
\end{array}\right) .
\end{aligned}
$$

It follows that the repeating unit is described by

$$
T_{2} T_{1}=\left(\begin{array}{cccc}
\frac{1}{\beta_{2}^{2}}\left(E^{2}+\beta_{1}^{2} e^{-2 i \lambda}\right)-1 & -\frac{E \beta_{1}}{\beta_{2}^{2}}\left(1+e^{-2 i \lambda}\right) & \frac{\beta_{1}}{\beta_{2}} e^{-2 i \lambda} & \frac{-E}{\beta_{2}} \\
-\frac{E \beta_{1}}{\beta_{2}^{2}}\left(1+e^{2 i \lambda}\right) & \frac{1}{\beta_{2}^{2}}\left(E^{2}+\beta_{1}^{2} e^{2 i \lambda}\right)-1 & -\frac{E}{\beta_{2}} & \frac{\beta_{1}}{\beta_{2}} e^{2 i \lambda} \\
-\frac{\beta_{1}}{\beta_{2}} & \frac{E}{\beta_{2}} & -1 & 0 \\
\frac{E}{\beta_{2}} & -\frac{\beta_{1}}{\beta_{2}} & 0 & -1
\end{array}\right) .
$$

For isolated armchair tubes, under the substitution

$$
E=-\sigma_{\mu} \sqrt{\beta_{1}^{2}+4 \beta_{1} \beta_{2} \cos \frac{\varphi}{2} \cos \lambda+4 \beta_{2}^{2} \cos ^{2} \frac{\varphi}{2}},
$$

and using Eqs. (4) and (C8), the diagonal matrix $T_{D}$ is expressed as

$$
T_{D}=\left(\begin{array}{cccc}
e^{i \varphi_{1}} & 0 & 0 & 0 \\
0 & e^{i \varphi_{2}} & 0 & 0 \\
0 & 0 & e^{-i \varphi_{1}} & 0 \\
0 & 0 & 0 & e^{-i \varphi_{2}}
\end{array}\right)
$$

with

$$
\begin{aligned}
& \cos \frac{\varphi_{1}}{2}=\frac{1}{2\left|\beta_{2}\right|}\left(-\left|\beta_{1}\right| \cos \lambda-\sqrt{E^{2}-\beta_{1}^{2} \sin ^{2} \lambda}\right), \\
& \cos \frac{\varphi_{2}}{2}=\frac{1}{2\left|\beta_{2}\right|}\left(-\left|\beta_{1}\right| \cos \lambda+\sqrt{E^{2}-\beta_{1}^{2} \sin ^{2} \lambda}\right),
\end{aligned}
$$

and via construction of the diagonalizing matrix $S,{ }^{17}$ we find, for $\left(T_{2} T_{1}\right)^{r}$, 


$$
\left(T_{2} T_{1}\right)^{r}=\left(\begin{array}{cccc}
-R_{1}(2 r+1) & -e^{-i \lambda} S(2 r+1) & -e^{-i \lambda} R_{1}(2 r) & -S(2 r) \\
-e^{i \lambda} S(2 r+1) & -R_{2}(2 r+1) & -S(2 r) & -e^{i \lambda} R_{2}(2 r) \\
e^{i \lambda} R_{1}(2 r) & S(2 r) & R_{1}(2 r-1) & e^{i \lambda} S(2 r-1) \\
S(2 r) & e^{-i \lambda} R_{2}(2 r) & e^{-i \lambda} S(2 r-1) & R_{2}(2 r-1)
\end{array}\right),
$$

with

$$
\begin{aligned}
R_{1}(x)=-\frac{1}{2}\left(\frac{\sin \left(x \frac{\varphi_{1}}{2}\right)}{\sin \left(\frac{\varphi_{1}}{2}\right)}+\frac{\sin \left(x \frac{\varphi_{2}}{2}\right)}{\sin \left(\frac{\varphi_{2}}{2}\right)}\right) \\
+i \frac{\beta_{1}}{E} S(x) \sin \lambda, \\
R_{2}(x)=-\frac{1}{2}\left(\frac{\sin \left(x \frac{\varphi_{1}}{2}\right)}{\sin \left(\frac{\varphi_{1}}{2}\right)}+\frac{\sin \left(x \frac{\varphi_{2}}{2}\right)}{\sin \left(\frac{\varphi_{2}}{2}\right)}\right) \\
-i \frac{\beta_{1}}{E} S(x) \sin \lambda,
\end{aligned}
$$

$$
S(x)=-\frac{E}{2 \beta_{1} \sqrt{\left(\frac{E}{\beta_{1}}\right)^{2}-\sin ^{2} \lambda}}
$$

$$
\times\left(\frac{\sin \left(x \frac{\varphi_{1}}{2}\right)}{\sin \left(\frac{\varphi_{1}}{2}\right)}-\frac{\sin \left(x \frac{\varphi_{2}}{2}\right)}{\sin \left(\frac{\varphi_{2}}{2}\right)}\right),
$$

and similar expressions for $\left(T_{1} T_{2}\right)^{r}, T_{1}\left(T_{2} T_{1}\right)^{r}$, and $\left(T_{2} T_{1}\right)^{r} T_{2}$. The following determinant of $E-\mathcal{H}_{\mathrm{A}}(\lambda)$ is found:

$$
\begin{aligned}
& D_{\mathrm{A}}(\lambda, N)=(-1)^{N} \beta_{2}^{2 N} \frac{\sin \left((N+1) \frac{\varphi_{1}}{2}\right)}{\sin \left(\frac{\varphi_{1}}{2}\right)} \\
& \times \frac{\sin \left((N+1) \frac{\varphi_{2}}{2}\right)}{\sin \left(\frac{\varphi_{2}}{2}\right)} .
\end{aligned}
$$

The eigenvalue problem can be solved exactly:

$$
\varphi_{1,2}=\frac{2 r \pi}{N+1}, \quad r=1, \ldots, N .
$$

Note that these solutions are contained in the spectrum of a polyhex carbon torus with indices $(n, n, N+1,-(N+1)){ }^{25}$ The coefficients of the wave functions can be written as

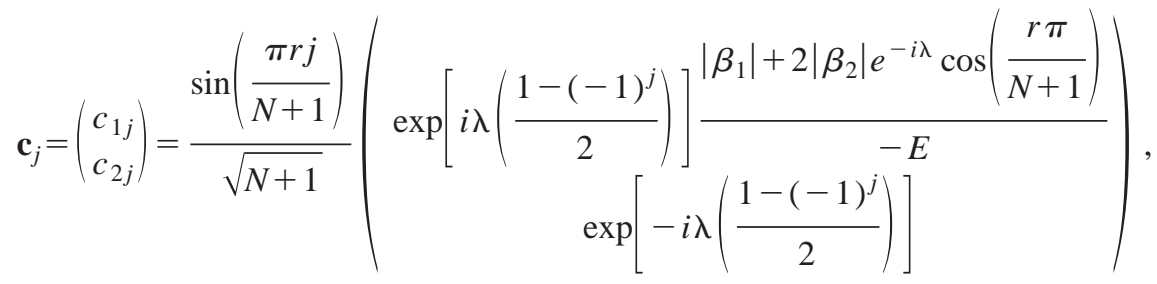

with $c_{1 j}$ and $c_{2 j}$ the coefficients of the orbitals at, respectively, the black and white atoms at ring $j$. All $\varphi$ are real: there are no decaying wave functions. The spectrum around energy zero is determined by $\mathcal{H}_{\mathrm{A}}(\lambda=0)$, where the coefficients have the simple form

$$
c_{2 j}=\sigma_{\mu} c_{1 j}=\frac{1}{\sqrt{N+1}} \sin \left(\frac{\pi r j}{N+1}\right),
$$

which is very much like the solutions of a $1 \mathrm{D}$ quantum box. ${ }^{4}$ Without curvature effects, the tube is a conductor for 
lengths $N=3 q-1$ and a semiconductor otherwise (see Appendix B). The band gap is equal to

$$
\begin{aligned}
& \Delta E_{N=3 q-1}=2|\beta|\left|1+2 \cos \left(\frac{2 q \pi}{3 q}\right)\right|=0, \\
& \Delta E_{N=3 q}=2|\beta|\left|1+2 \cos \left(\frac{(2 q+1) \pi}{3 q+1}\right)\right| \\
& \approx \frac{2|\beta| \pi}{3}\left(\frac{1}{\sqrt{3} q}-\frac{(6 \sqrt{3}+\pi)}{54 q^{2}}\right), \\
& \Delta E_{N=3 q+1}=2|\beta|\left|1+2 \cos \left(\frac{(2 q+1) \pi}{3 q+2}\right)\right| \\
& \approx \frac{2|\beta| \pi}{3}\left(\frac{1}{\sqrt{3} q}-\frac{(12 \sqrt{3}-\pi)}{54 q^{2}}\right) .
\end{aligned}
$$

This indicates a band-gap minimum for $N=3 q-1$ tubes and almost equal band gaps for $N=3 q$ and $N=3 q+1$ tubes. However, electronic structure calculations in Refs. 11 and 14 clearly show an oscillation of the band-gap maximum-each time at $N=3 q+1$-but the pattern followed by the band-gap minimum is less clear. Liu et al. ${ }^{14}$ use the presence or absence of an alternating double-bond configuration to explain this intuitively. We will show here that it can be explained mathematically with a simple tight-binding model, if one includes curvature. Two key elements are common to both models: the finiteness of the system and the destruction of the threefold symmetry of the parent graphite plane. The derivations are proven in Appendix B. The HOMO-LUMO gap is given by the following formula:

$$
\Delta E=2\left|\beta_{1}+2 \beta_{2} \cos \left(\frac{2 \pi}{3}+\frac{\pi}{N+1}(\kappa-h)\right)\right|,
$$

where

$$
\kappa=\operatorname{Int}\left[\frac{\Delta \varphi_{F}}{2 \pi}(N+1)+h\right],
$$

with $\operatorname{Int}[x]$ the integer closest to $x, h$ equal to

$$
\begin{aligned}
& h=0, \quad \text { for } N=3 q-1, \\
& h=\frac{2}{3}, \quad \text { for } N=3 q, \\
& h=\frac{1}{3}, \quad \text { for } N=3 q+1,
\end{aligned}
$$

and $\Delta \varphi_{F}$ the shift of the Fermi point due to curvature:

$$
\Delta \varphi_{F}=-\frac{4 \pi}{3}+2 \arccos \left(-\frac{\beta_{1}}{2 \beta_{2}}\right) \approx \frac{\Delta \beta}{\sqrt{3} \beta},
$$

with

$$
\Delta \beta=\beta_{1}-\beta_{2} .
$$

Equation (65) is slightly approximative around the maxima, since it is built on the approximation that $E\left(\varphi_{F}-\Delta x\right)$ $=E\left(\varphi_{F}+\Delta x\right)$. However, in Fig. 5 there is a perfect agreement between the exact calculated points and Eq. (65) where in the latter case $N$ is varied continuously. The inclusion of curvature opens a HOMO-LUMO gap for $N=3 q-1$ tubes.
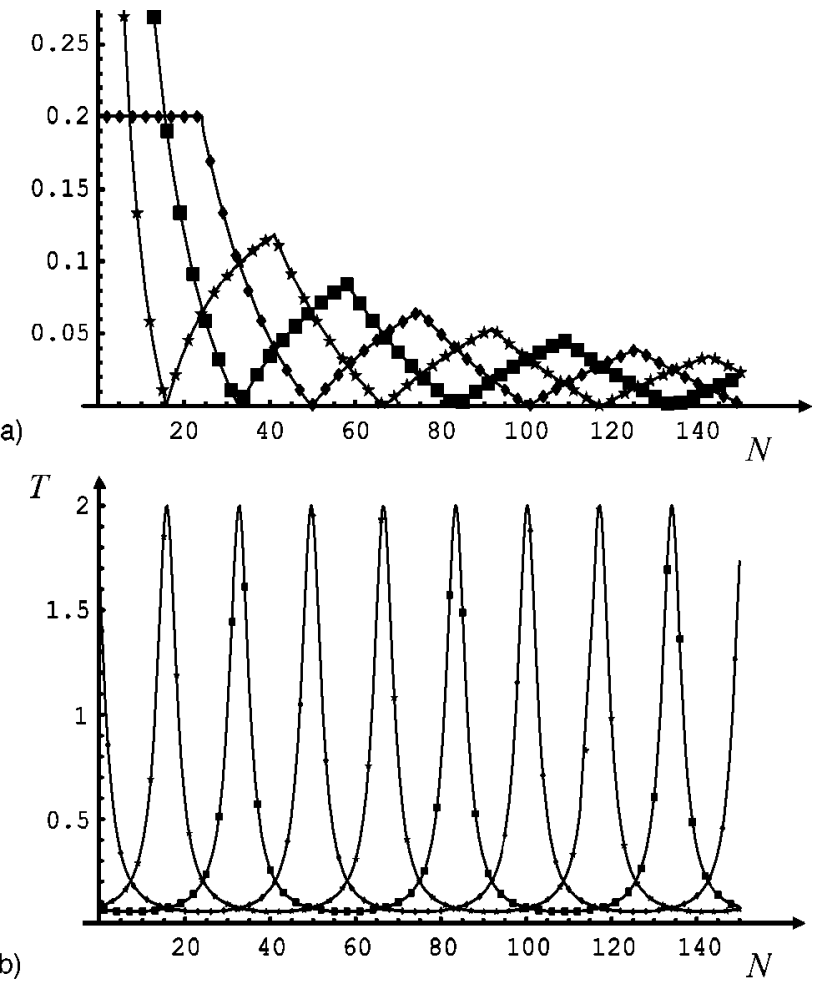

FIG. 5. (a) HOMO-LUMO gap and (b) transmission at the Fermi level of the armchair nanotube versus length for $N=3 q-1$ (diamonds), $N=3 q$ (stars), and $N=3 q+1$ (squares). The hopping parameters are taken as $\beta_{1}$ $=1.05$ and $\beta_{2}=0.95$. The coupling is taken as $\Delta_{1}=0.1, \Delta_{2}=0$.

The gap of these tubes stays first constant with length at a value $2\left|\beta_{1}-\beta_{2}\right|$ (we suppose $\left|\beta_{1}\right|>\left|\beta_{2}\right|$ ), and then starts to decrease when

$$
N+1>\frac{\pi}{\left|\Delta \varphi_{F}\right|}, \quad \text { for } N=3 q-1 .
$$

The HOMO-LUMO gap for the other two cases falls below $2\left|\beta_{1}-\beta_{2}\right|$ at an earlier stage. The condition for $\Delta E<2 \mid \beta_{1}$ $-\beta_{2} \mid$ is for the three tubes:

$$
N+1>\frac{\pi}{\Delta \varphi_{F}}(1-h) \text {. }
$$

So the HOMO-LUMO gap for $N=3 q$ and $N=3 q+1$ tubes becomes smaller than that for $N=3 q-1$ tubes at, respectively, one-third and two-thirds of the length where the gap of the $N=3 q-1$ tubes begins to decrease. The first crossing point of the band gap for $N=3 q$ tubes with that of $N=3 q$ -1 tubes is indeed visible in Fig. 1 of Ref. 11 and in Fig. 3 of Ref. 14. The second crossing point is outside the range of these figures, but seems to be reached at approximately twice the length of the first crossing point if one extrapolates to larger lengths. At even larger lengths one encounters a damped oscillatory behavior in function of length for the three kinds of tubes separately as one can see in Fig. 5(a). Let us vary $N$ continuously. The band gap will drop to zero each time a point $\varphi=2 \pi / 3+[\pi /(N+1)](\kappa-h)$ falls on the Fermi point $\varphi_{F}$. This happens when

$$
N+1=\frac{2 \pi}{\Delta \varphi_{F}}(\kappa-h), \quad \kappa=1,2, \ldots .
$$


A maximum is reached whenever two neighboring points are at the same distance from the Fermi point. This gives the following condition:

$$
N+1=\frac{2 \pi}{\Delta \varphi_{F}}\left(\kappa-h+\frac{1}{2}\right), \quad \kappa=1,2, \ldots .
$$

It is at the position of the maxima that $\kappa$ increases by 1 , so at this position there is a switching of the wave function. The period of oscillation is

$$
\Delta N=\frac{2 \pi}{\Delta \varphi_{F}} .
$$

The height of the maxima is, to first order,

$$
\Delta E_{\max }=\frac{\pi \sqrt{3} \beta}{N_{\max }+1},
$$

so is inversely proportional with tube length. The position of the minima, maxima, and height of the maxima are shown in Table I.

\section{TRANSMISSION}

\section{A. Zigzag tubes}

Applying Eq. (13), the transmission $\mathcal{T}$ can be divided into a sum of terms $\mathcal{T}(\lambda)$, each belonging to a different matrix block $\mathcal{H}(\lambda)$, as long as the leads do not lower the rotational symmetry of the system,
TABLE I. The behavior of the HOMO-LUMO gap of armchair tubes with length.

\begin{tabular}{cccc}
\hline \hline Class & $\Delta E<2\left|\beta_{1}-\beta_{2}\right|$ & $\Delta E \approx 0^{\mathrm{a}}$ & $\Delta E \approx \Delta E_{\max } \mathrm{a}^{\mathrm{a}}$ \\
\hline$N=3 q-1$ & $N+1<\frac{\pi}{\Delta \varphi_{F}}$ & $N+1 \approx \frac{2 \kappa \pi}{\Delta \varphi_{F}}$ & $N+1 \approx \frac{2 \pi}{\Delta \varphi_{F}}\left(\kappa+\frac{1}{2}\right)$ \\
$N=3 q$ & $N+1<\frac{\pi}{3 \Delta \varphi_{F}}$ & $N+1 \approx \frac{2 \pi}{\Delta \varphi_{F}}\left(\kappa-\frac{2}{3}\right)$ & $N+1 \approx \frac{2 \pi}{\Delta \varphi_{F}}\left(\kappa-\frac{1}{6}\right)$ \\
$N=3 q+1$ & $N+1<\frac{2 \pi}{3 \Delta \varphi_{F}}$ & $N+1 \approx \frac{2 \pi}{\Delta \varphi_{F}}\left(\kappa-\frac{1}{3}\right)$ & $N+1 \approx \frac{2 \pi}{\Delta \varphi_{F}}\left(\kappa+\frac{1}{6}\right)$
\end{tabular}

${ }^{\mathrm{a}} \boldsymbol{\kappa}=1,2, \ldots$.

$$
\mathcal{T}=\sum_{\lambda} \mathcal{T}(\lambda)
$$

and due to symmetry reasons,

$$
\mathcal{T}(\lambda)=\mathcal{T}(-\lambda) \text {. }
$$

In the case of zigzag tubes, the matrix $Q$ becomes completely diagonalized, so each $\mathcal{T}(\lambda)$ corresponds to one individual channel and is a number between 0 and 1 . The determinant of a system with leads in the case of a HH tube can be written as

$$
\widetilde{D}_{\mathrm{Z}}^{\mathrm{HH}}(\lambda, N)=\left|\begin{array}{cccccc}
E-\Sigma_{11}(\lambda) & -2 \beta_{2} \cos \lambda & 0 & & & \\
-2 \beta_{2} \cos \lambda & E & -\beta_{1} & & & \\
0 & -\beta_{1} & \cdots & & & \\
& & & \ldots & -\beta_{1} & 0 \\
& & & -\beta_{1} & E & -2 \beta_{2} \cos \lambda \\
& & & 0 & -2 \beta_{2} \cos \lambda & E-\Sigma_{N N}(\lambda)
\end{array}\right|,
$$

which with simple determinant methods can be proven to be

$$
\widetilde{D}_{\mathrm{Z}}^{\mathrm{HH}}(\lambda, N)=D_{\mathrm{Z}}^{\mathrm{HH}}(\lambda, N)-\left[\Sigma_{11}(\lambda)+\Sigma_{N N}(\lambda)\right] D_{\mathrm{Z}}^{\mathrm{HM}}(\lambda, N-1)+\Sigma_{11}(\lambda) \Sigma_{N N}(\lambda) D_{\mathrm{Z}}^{\mathrm{MM}}(\lambda, N-2),
$$

and similar expressions hold for $\widetilde{D}_{\mathrm{Z}}^{\mathrm{MM}}(\lambda, N)$ and $\widetilde{D}_{\mathrm{Z}}^{\mathrm{HM}}(\lambda, N)$. This method was also used in the case of a simple linear chain in Ref. 26. For simplicity, we take identical leads, take the same parameter irrespective of $\lambda$, and assume that it is constant and purely imaginary. ${ }^{23}$ So the self-energy terms become

$$
\Sigma_{11}(\lambda)=\Sigma_{N N}(\lambda)=-i \Delta \text {. }
$$

A dependence of the self-energy on $\lambda$ and on the energy can be made explicit only when a certain model for the leads is assumed. In that case, the formula following hereafter can be easily adapted. The dependence of the self-energy on $\lambda$ is actually only important when calculating the total transmission-i.e., when adding contributions of different rotational symmetry. Using Eqs. (4), (12), (14), (77), and (78) for the transmission we obtain the following expression for zigzag tubes (see Appendix C):

$$
\begin{aligned}
& \mathcal{T}_{\mathrm{Z}}^{\mathrm{XX}}(\lambda, N)=\left|\frac{2 \Delta(-1)^{N+1} b_{1}^{N / 2} b_{2}^{N / 2-1}}{D_{\mathrm{Z}}^{\mathrm{XX}}(\lambda, N)+2 i \Delta D_{\mathrm{Z}}^{\mathrm{HM}}(\lambda, N-1)-\Delta^{2} D_{\mathrm{Z}}^{\mathrm{YY}}(\lambda, N-2)}\right|^{2}, \\
& \mathcal{T}_{\mathrm{Z}}^{\mathrm{HM}}(\lambda, N)=\left|\frac{2 \Delta(-1)^{N+1}\left(b_{1} b_{2}\right)^{(N-1) / 2}}{D_{\mathrm{Z}}^{\mathrm{HM}}(\lambda, N)+i \Delta\left[D_{\mathrm{Z}}^{\mathrm{HH}}(\lambda, N-1)+D_{\mathrm{Z}}^{\mathrm{MM}}(\lambda, N-1)\right]-\Delta^{2} D_{\mathrm{Z}}^{\mathrm{HM}}(\lambda, N-2)}\right|^{2},
\end{aligned}
$$


where $b_{1}=2 \beta_{2} \cos \lambda$ and $b_{2}=\beta_{1}$ for $\mathrm{XX}=\mathrm{HH}, \mathrm{YY}=\mathrm{MM}$, and vice versa for $X X=M M, Y Y=H H$. When the transmission is plotted versus the energy, one obtains a series of peaks. The transmission is high when the energy is in resonance with one of the scattering states and low when it is between two scattering states, and for low coupling the scattering states are close to the eigenstates of the isolated system. In Ref. 16 it was found numerically that the transmission of finite semiconducting tubes shows a resonant peak at the Fermi level that decays with length. In the following, we explain the origin of this peak.

At energy zero Eq. (79) becomes

$$
\begin{aligned}
& \mathcal{T}_{\mathrm{Z}}^{\mathrm{XX}}(\lambda, N)=\frac{4}{2+\frac{b_{1}^{N-1}}{b_{2}^{N-1}} \frac{b_{1} b_{2}}{\Delta^{2}}+\frac{b_{2}^{N-1}}{b_{1}^{N-1}} \frac{\Delta^{2}}{b_{1} b_{2}}}, \\
& \mathcal{T}_{\mathrm{Z}}^{\mathrm{HM}}(\lambda, N)=\frac{4}{2+\frac{b_{1}^{N-1}}{b_{2}^{N-1}}+\frac{b_{2}^{N-1}}{b_{1}^{N-1}}} \approx 4\left|\frac{\beta_{1}}{2 \beta_{2} \cos \lambda}\right|^{s(N-1)},
\end{aligned}
$$

where $s=1$ for $\beta_{1}<2 \beta_{2} \cos \lambda$ and $s=-1$ for $\beta_{1}$ $>2 \beta_{2} \cos \lambda$. Figures 6(a) and 6(b) show some examples of $\mathcal{T}_{\mathrm{Z}}^{\mathrm{XY}}(\lambda, N)$ as a function of length for different $\lambda$ at energy zero. One can see from Eqs. (80) that $\mathcal{T}_{\mathrm{Z}}^{\mathrm{HM}}(\lambda, N)$ at the Fermi level, as opposed to $\mathcal{T}_{\mathrm{Z}}^{\mathrm{HH}}(\lambda, N)$ and $\mathcal{T}_{\mathrm{Z}}^{\mathrm{MM}}(\lambda, N)$, is independent of the coupling strength of the leads. When $N$ is treated as a continuous variable, it is possible to rewrite $\mathcal{T}_{\mathrm{Z}}^{\mathrm{XX}}(\lambda, N)$ :

$$
\begin{aligned}
& \mathcal{T}_{Z}^{\mathrm{XX}}(\lambda, N)=\frac{4}{2+\frac{b_{1}^{N^{\prime}-1}}{b_{2}^{N^{\prime}-1}}+\frac{b_{2}^{N^{\prime}-1}}{b_{1}^{N^{\prime}-1}},} \\
& N^{\prime}=N+1-\frac{\ln \left|\frac{\Delta^{2}}{b_{1}^{2}}\right|}{\ln \left|\frac{b_{2}}{b_{1}}\right|},
\end{aligned}
$$

so $\mathcal{T}_{\mathrm{Z}}^{\mathrm{XX}}(\lambda, N)$ is identical to $\mathcal{T}_{\mathrm{Z}}^{\mathrm{HM}}(\lambda, N)$ except that there is a shift of the curve. It follows that the coupling can influence the position of the curve $\mathcal{T}_{\mathrm{Z}}^{\mathrm{XX}}(\lambda, N)$ but not the shape. $\mathcal{T}_{\mathrm{Z}}^{\mathrm{HH}}(\lambda, N)$ and $\mathcal{T}_{\mathrm{Z}}^{\mathrm{MM}}(\lambda, N)$ are displaced with the same distance from $\mathcal{T}_{\mathrm{Z}}^{\mathrm{HM}}(\lambda, N)$, but in opposite directions. By solving $\partial \mathcal{T}_{\mathrm{Z}}^{\mathrm{XY}}(\lambda, N) / \partial N=0$ we find that the transmission is maximal and unity when

$$
\begin{aligned}
& N=1, \text { for HM, } \\
& N=\frac{\ln \left|\frac{\Delta^{2}}{b_{1}^{2}}\right|}{\ln \left|\frac{b_{2}}{b_{1}}\right|}, \text { for } \mathrm{HH}, \mathrm{MM},
\end{aligned}
$$

and decreases exponentially when deviating from this maximum. The decrease is faster when $b_{2} / b_{1}$ deviates more from unity. Of course $N$ is actually a nonzero natural number. So $\mathcal{T}_{\mathrm{Z}}^{\mathrm{HM}}(\lambda, N)$ starts at unity and decreases exponentially for bigger $N$, as can be seen in Figs. 6(a) and 6(b). Here
$\mathcal{T}_{\mathrm{Z}}^{\mathrm{XX}}(\lambda, N)$ starts at a lower value, rises first to a maximum, and then decreases again if the following condition is fulfilled:

$$
\frac{\Delta-\sqrt{b_{2} b_{1}}}{\left|b_{2}\right|-\left|b_{1}\right|}>0
$$

which is the case for $\mathcal{T}_{\mathrm{Z}}^{\mathrm{MM}}(\pi / 6, N)$ in Fig. 6(a) and $\mathcal{T}_{\mathrm{Z}}^{\mathrm{HH}}(\pi / 3, N)$ in Fig. 6(b). If Eq. (83) is not fulfilled, $\mathcal{T}_{\mathrm{Z}}^{\mathrm{XX}}(\lambda, N)$ decreases immediately and will not contribute much to the total transmission, which is the case for $\mathcal{T}_{\mathrm{Z}}^{\mathrm{HH}}(\pi / 6, N)$ in Fig. 6(a) and $\mathcal{T}_{\mathrm{Z}}^{\mathrm{MM}}(\pi / 3, N)$ in Fig. 6(b). An important note is that it is in principle possible to tune the coupling to have a maximal transmission for a given length.

The connection between the decay of the wave function and the decay of the transmission at zero energy in the case

a $T(\pi / 6)$

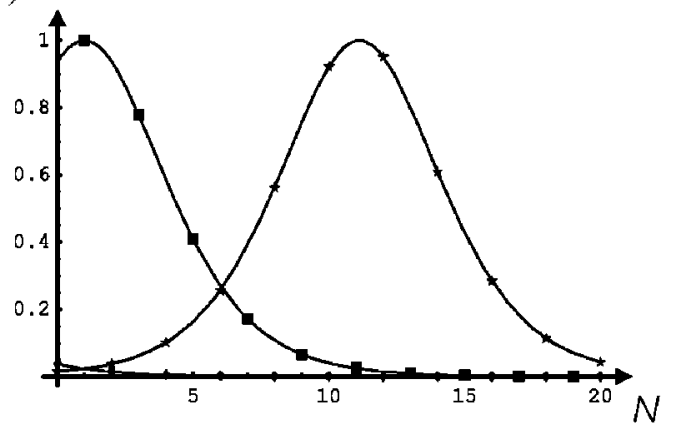

b
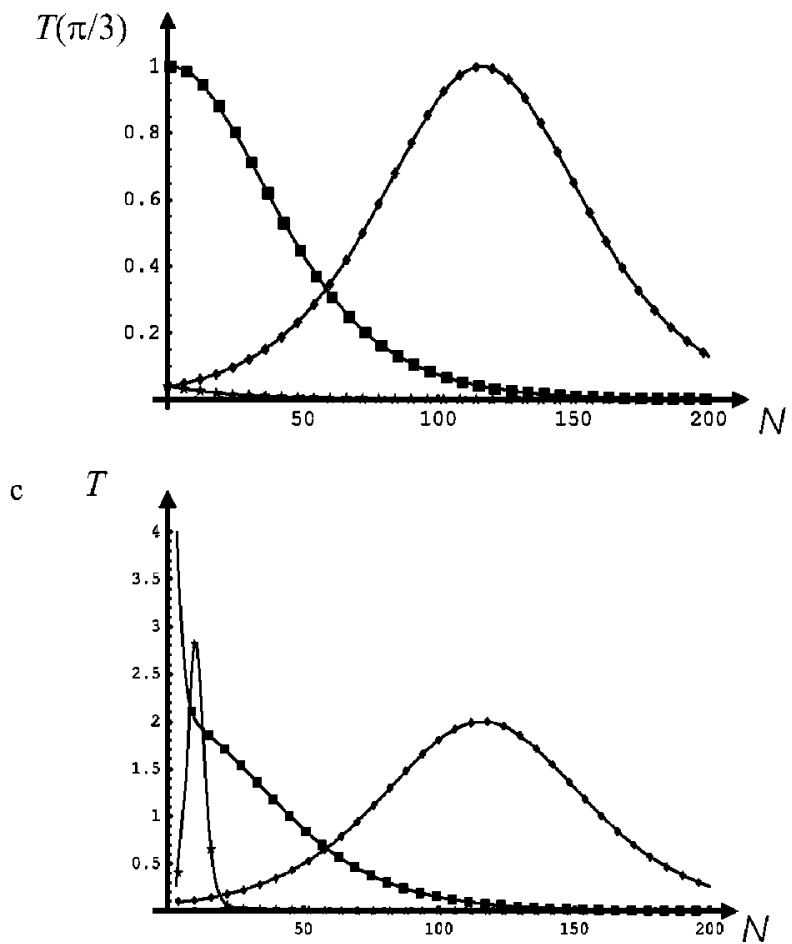

FIG. 6. Transmission at the Fermi level in the case of a HH (diamonds), a MM (stars), and a HM (squares) zigzag tube. (a) The contribution $\mathcal{T}(\pi / 6)$, (b) the contribution $\mathcal{T}(\pi / 3)$, and (c) the total transmission of a $(6,0)$ tube for $N>3$. The hopping parameters are taken as $\beta_{1}=\beta_{2}=1$. The coupling is taken as $\Delta=0.1$ for all the channels. 
of HM tubes can also be made: while the amplitude of the wave function has decreased by a factor $\left(2 \beta_{2} \cos \lambda / \beta_{1}\right)^{s(N-1) / 2}$ from edge to edge, $\mathcal{T}_{\mathrm{Z}}^{\mathrm{HM}}(\lambda, N)$ has dropped by a factor $\left(2 \beta_{2} \cos \lambda / \beta_{1}\right)^{s(N-1)}$. This connection between wave function and transmission can be made explicit with the help of Green's function. The tight-binding retarded Green's function of an isolated system is equal to

$$
G_{i j}^{0}(z)=\sum_{k} \frac{c_{i}^{k} c_{j}^{k *}}{z-E_{k}},
$$

with $z=E+i 0^{+}, E_{k}$ the eigenenergies of the system and $c_{i}^{k}, c_{j}^{k}$ the coefficients of the $k$ th wave function on the atoms $i$ and $j$, respectively. In the case of the system $\mathcal{H}_{\mathrm{Z}}^{\mathrm{HM}}(\lambda)$ the Green's function becomes, via Eqs. (38) and (39),

$$
\begin{aligned}
& G_{11}^{0}(z)=\frac{\left(\frac{2 \beta_{2} \cos \lambda}{\beta_{1}}\right)^{2}-1}{\left(\frac{2 \beta_{2} \cos \lambda}{\beta_{1}}\right)^{N+1}-1} \frac{1}{z}+\frac{2}{N+1} \sum_{\mu, f} \frac{\left[\sin \left(f \frac{2 \pi}{N+1}\right) 2 \beta_{2} \cos \lambda\right]^{2}}{E_{f}^{2}\left(z-E_{\mu f}\right)} \\
& =\frac{\left(\frac{2 \beta_{2} \cos \lambda}{\beta_{1}}\right)^{2}-1}{\left(\frac{2 \beta_{2} \cos \lambda}{\beta_{1}}\right)^{N+1}-1} \frac{1}{z}+\frac{4 z}{N+1} \sum_{f} \frac{\left[\sin \left(f \frac{2 \pi}{N+1}\right) 2 \beta_{2} \cos \lambda\right]^{2}}{E_{f}^{2}\left(z^{2}-E_{f}^{2}\right)}, \\
& G_{N N}^{0}(z)=\frac{\left(\frac{2 \beta_{2} \cos \lambda}{\beta_{1}}\right)^{2}-1}{\left(\frac{2 \beta_{2} \cos \lambda}{\beta_{1}}\right)^{N+1}-1} \frac{\left(-\frac{2 \beta_{2} \cos \lambda}{\beta_{1}}\right)^{N-1}}{z}+\frac{4 z}{N+1} \sum_{f} \frac{\left[\sin \left((N-1) f \frac{\pi}{N+1}\right) \beta_{1}\right]^{2}}{E_{f}^{2}\left(z^{2}-E_{f}^{2}\right)}, \\
& G_{1 N}^{0}(z)=\frac{\left(\frac{2 \beta_{2} \cos \lambda}{\beta_{1}}\right)^{2}-1}{\left(\frac{2 \beta_{2} \cos \lambda}{\beta_{1}}\right)^{N+1}-1} \frac{\left(-\frac{2 \beta_{2} \cos \lambda}{\beta_{1}}\right)^{(N-1) / 2}}{z}+\frac{4 z}{N+1} \\
& \times \sum_{f} \frac{e^{[(N-1) / 2] i \lambda} \sin \left(f \frac{2 \pi}{N+1}\right) \sin \left((N-1) f \frac{\pi}{N+1}\right) 2 \beta_{1} \beta_{2} \cos \lambda}{E_{f}^{2}\left(z^{2}-E_{f}^{2}\right)}=G_{N 1}^{0}(z) .
\end{aligned}
$$

When $E \rightarrow 0$, the first term in each function of Eqs. (85), corresponding to a decaying eigenstate of the tube, becomes infinite while the second term is going to zero. In other words, at the Fermi level the Green's function and as a consequence all properties derived from it are solely determined by the decaying wave function. The Green's function of the system with leads can be calculated from the Green's function of the isolated system making use of Dyson's equation $^{27,28}$

$$
G=\left(I-G^{0} \Sigma\right)^{-1} G^{0},
$$

so we get

$$
G_{1 N}=\frac{G_{1 N}^{0}}{-1+\Sigma\left(G_{11}^{0}+G_{N N}^{0}\right)-\Sigma^{2}\left(\left(G_{1 N}^{0}\right)^{2}-G_{11}^{0} G_{N N}^{0}\right)} .
$$

At $E=0,\left(G_{1 N}^{0}\right)^{2}$ and $G_{11}^{0} G_{N N}^{0}$ cancel out and we get

$$
G_{1 N}=\frac{z G_{1 N}^{0}}{\sum_{z}\left(G_{11}^{0}+G_{N N}^{0}\right)}=-\frac{\left(-\frac{2 \beta_{2} \cos \lambda}{\beta_{1}}\right)^{(N-1) / 2}}{-i \Delta\left[1+\left(-\frac{2 \beta_{2} \cos \lambda}{\beta_{1}}\right)^{N-1}\right]},
$$

which via Eqs. (12) allows us to recover the previous result

$$
\begin{aligned}
\mathcal{T}^{\mathrm{HM}}(\lambda, N) & =4 \Delta^{2}\left|G_{1 N}\right|^{2} \\
& =\frac{4}{2+\left|\frac{\beta_{1}}{2 \beta_{2} \cos \lambda}\right|^{N-1}+\left|\frac{2 \beta_{2} \cos \lambda}{\beta_{1}}\right|^{N-1}} .
\end{aligned}
$$

So it is proved that the transmission at the Fermi level for HM tubes is only determined by the non bonding decaying wave functions.

The channel contribution that decays most slowly with $N$ and which survives longest at larger lengths is $\mathcal{T}_{\mathrm{Z}}(\pi / 3, N)$, for which Eq. (80) becomes 


$$
\begin{aligned}
& \mathcal{T}_{\mathrm{Z}}^{\mathrm{HH}}\left(\frac{\pi}{3}, N\right)=\frac{4}{2+\frac{\beta_{2}^{N}}{\beta_{1}^{N-2} \Delta^{2}}+\frac{\beta_{1}^{N-2} \Delta^{2}}{\beta_{2}^{N}}} \approx \frac{4}{2+\frac{\beta^{2}}{\Delta^{2}}+\frac{\Delta^{2}}{\beta^{2}}}, \\
& \mathcal{T}_{\mathrm{Z}}^{\mathrm{MM}}\left(\frac{\pi}{3}, N\right)=\frac{4}{2+\frac{\beta_{1}^{N}}{\beta_{2}^{N-2} \Delta^{2}}+\frac{\beta_{2}^{N-2} \Delta^{2}}{\beta_{1}^{N}}} \approx \frac{4}{2+\frac{\beta^{2}}{\Delta^{2}}+\frac{\Delta^{2}}{\beta^{2}}},
\end{aligned}
$$

$\mathcal{T}_{\mathrm{Z}}^{\mathrm{HM}}\left(\frac{\pi}{3}, N\right)=\frac{4}{2+\left|\frac{\beta_{1}}{\beta_{2}}\right|^{N-1}+\left|\frac{\beta_{2}}{\beta_{1}}\right|^{N-1}} \approx 1$.

The approximate expressions here are obtained for $\beta_{1}$ $\approx \beta_{2}$. It becomes clear from this equation that neglect of curvature leads to a transmission independent of $N$, although the width of the peak becomes smaller as $N$ increases. So for bigger diameters (smaller curvature effects) $\mathcal{T}(\pi / 3, N)$ and as consequence the total transmission at the Fermi level will decay less rapidly with length. The same conclusion has been reached for side-contacted infinite nanotubes. ${ }^{28}$

We had seen before that for the three kinds of tubes, with $n$ even, there was a high degeneracy at energy $\pm \beta_{1}$, due to the eigenvalues of $\mathcal{H}_{\mathrm{Z}}(\pi / 2)$. The term $\mathcal{T}_{\mathrm{Z}}(\pi / 2, N)$ becomes

$$
\mathcal{T}_{\mathrm{Z}}^{\mathrm{HM}}\left(\frac{\pi}{2}, N>1\right)=\mathcal{T}_{\mathrm{Z}}^{\mathrm{HH}}\left(\frac{\pi}{2}, N>0\right)=\mathcal{T}_{\mathrm{Z}}^{\mathrm{MM}}\left(\frac{\pi}{2}, N>2\right)=0
$$

even for $E= \pm \beta_{1}$. The reason for this is that, since $2 \beta_{2} \cos (\lambda)=0$, the linear graph in Fig. 3(a) is no longer fully connected, so there is no bridge anymore between the two leads. We note in passing that the breaking of the chain in Fig. 3(a) into isolated dimers explains the multifold degeneracy of the corresponding eigenvalues $\pm \beta_{1}$. For the same reason, the eigenstates corresponding to $E=0, \lambda=\pi / 2$ [one for $(2 q, N, \mathrm{HM})$ tubes, two for $(2 q, N, \mathrm{HH})$ tubes, none for $(2 q, N, \mathrm{MM})$ tubes] cannot contribute to the transmission along the nanotube axis. In other words, although these states are at the Fermi level, they do not make a "metal" of the tube, as opposed to what is suggested in, e.g., Ref. 10. In Fig. $6(\mathrm{c})$ the total transmission at the Fermi level as a function of length is given for a $(6, N, \mathrm{HM}),(6, N, \mathrm{HH})$, and $(6, N, \mathrm{MM})$ tube. For HM tubes, the transmission begins high due to the channels $\mathcal{T}_{\mathrm{Z}}^{\mathrm{HM}}(0, N), \mathcal{T}_{\mathrm{Z}}^{\mathrm{HM}}( \pm \pi / 6, N)$, and $\mathcal{T}_{\mathrm{Z}}^{\mathrm{HM}}( \pm \pi / 3, N)$, but at larger lengths only the contributions $\mathcal{T}_{\mathrm{Z}}^{\mathrm{HM}}( \pm \pi / 3, N)$ play a significant role. For MM tubes, we have a peak at short lengths that is close to three due to the terms $\mathcal{T}_{\mathrm{Z}}^{\mathrm{MM}}(0, N)$ and $\mathcal{T}_{\mathrm{Z}}^{\mathrm{MM}}( \pm \pi / 6, N)$, but afterwards the decay is very fast, since there is not much contribution from the terms $\mathcal{T}_{\mathrm{Z}}^{\mathrm{MM}}$ $( \pm \pi / 3, N)$. For $\mathrm{HH}$ tubes finally, the shape is almost totally determined by the terms $\mathcal{T}_{\mathrm{Z}}^{\mathrm{HH}}( \pm \pi / 3, N)$. The term $\mathcal{T}_{\mathrm{Z}}(\pi / 2, N)$ never contributes [except for the pathological cases $N=1$ and $N=2$, which are not included in Fig. 6(c)].

\section{B. Armchair tubes}

Also for the armchair tube the transmission can be divided into contributions $\mathcal{T}_{\mathrm{A}}(\lambda, N)$ with different rotational symmetry. The matrix $Q_{\mathrm{A}}$ is block-diagonalized into $2 \times 2$ matrices $Q_{\mathrm{A}}(\lambda, N)$, so each $\mathcal{T}_{\mathrm{A}}(\lambda, N)$ is a sum of two channels and as a consequence is a number between 0 and 2 . The self-energy matrices $\Sigma_{11}(\lambda)$ and $\Sigma_{N N}(\lambda)$ are now $2 \times 2$ matrices instead of numbers as was the case for zigzag tubes. As we did for the zigzag tubes, we assume identical leads, the same parameter irrespective of $\lambda$, and that it is constant and purely imaginary: ${ }^{23}$

$$
\Sigma_{11}(\lambda)=\Sigma_{N N}(\lambda)=\left(\begin{array}{cc}
-i \Delta_{1} & -i \Delta_{2} \\
-i \Delta_{2} & -i \Delta_{1}
\end{array}\right)
$$

Then $\mathcal{T}_{\mathrm{A}}(\lambda, N)$ is equal to (see Appendix C)

$$
\mathcal{T}_{\mathrm{A}}(\lambda, N)=\frac{4\left\{\Delta_{1}^{2}\left(|a|^{2}+|c|^{2}+|b|^{2}+|d|^{2}\right)+\Delta_{2}^{2}\left(a d^{*}+b^{*} c+\text { c.c. }\right)-\Delta_{1} \Delta_{2}\left[\left(a^{*}+d^{*}\right)(b+c)+\text { c.c. }\right]\right\}}{|a d-b c|^{2}} .
$$

The coefficients of the matrix

$$
T_{11}=\left(\begin{array}{ll}
a & b \\
c & d
\end{array}\right)
$$

can be found in Appendix $\mathrm{C}$.

The graph of $\mathcal{H}_{\mathrm{A}}(0)$ forms a normal bilinear chain or ladder. By applying the symmetry of the mirror plane that is perpendicular to all $\beta_{1}$ bonds, $\mathcal{H}_{\mathrm{A}}(0)$ can be further block-diagonalized into $\mathcal{H}_{\mathrm{A}}^{+}(0)$ and $\mathcal{H}_{\mathrm{A}}^{-}(0)$. Their graphs correspond to that of a simple linear chain with hopping parameter $\beta_{2}$ and diagonal element plus and minus $\beta_{1}$ for $\mathcal{H}_{\mathrm{A}}^{+}(0)$ and $\mathcal{H}_{\mathrm{A}}^{-}(0)$, respectively, and are shown in Fig. 3(b). So according to Eq. (13) $\mathcal{T}_{\mathrm{A}}(0, N)$ can be divided into two individual channels $\mathcal{T}_{\mathrm{A}}^{+}(0, N)$ and $\mathcal{T}_{\mathrm{A}}^{-}(0, N)$ : 


$$
\begin{aligned}
& \mathcal{T}_{\mathrm{A}}(0, N)=\mathcal{T}_{\mathrm{A}}^{+}(0, N)+\mathcal{T}_{\mathrm{A}}^{-}(0, N), \\
& \mathcal{T}_{\mathrm{A}}^{ \pm}(0, N)=\left|\frac{2 \Delta^{ \pm}}{\beta_{2} \frac{\sin \left[(N+1) \theta^{ \pm}\right]}{\sin \theta^{ \pm}}+2 i \Delta^{ \pm} \frac{\sin \left(N \theta^{ \pm}\right)}{\sin \theta^{ \pm}}-\frac{\left(\Delta^{ \pm}\right)^{2}}{\beta_{2}} \frac{\sin \left[(N-1) \theta^{ \pm}\right]}{\sin \theta^{ \pm}}}\right|^{2},
\end{aligned}
$$

with $\theta^{ \pm}=\arccos \left[\left(E \mp \beta_{1}\right) / 2 \beta_{2}\right]$ and $\Delta^{ \pm}=\Delta_{1} \pm \Delta_{2}$. The relationship with $\varphi_{1}, \varphi_{2}$ defined in Eq. (58) is

$$
\cos \frac{\varphi_{1}}{2}=\mp \cos \theta^{\mp}, \quad \cos \frac{\varphi_{2}}{2}= \pm \cos \theta^{ \pm} .
$$

The upper sign is for $E / \beta_{2}>0$ and the lower for $E / \beta_{2}<0$. Also for $\mathcal{H}_{\mathrm{A}}(\pi / 2)$ this mirror symmetry can be applied to form linear chains with hopping parameter $\beta_{2}$ and diagonal element plus and minus $\beta_{1}$ on alternating atoms [see Fig. 2(b)]. Here $\mathcal{T}_{\mathrm{A}}(\pi / 2, N)$ becomes

$$
\mathcal{T}_{\mathrm{A}}\left(\frac{\pi}{2}, N\right)=\mathcal{T}_{\mathrm{A}}^{+}\left(\frac{\pi}{2}, N\right)+\mathcal{T}_{\mathrm{A}}^{-}\left(\frac{\pi}{2}, N\right) .
$$

The contributions $\mathcal{T}_{\mathrm{A}}^{+}(\pi / 2, N)$ and $\mathcal{T}_{\mathrm{A}}^{-}(\pi / 2, N)$ are, for $N$ even,

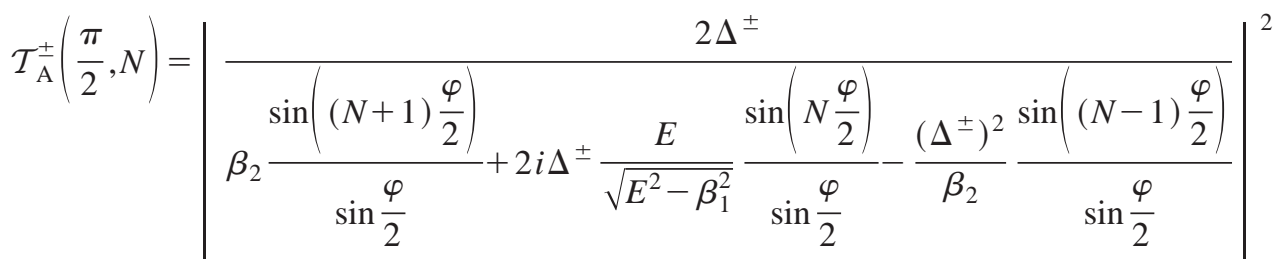

and, for $N$ odd,

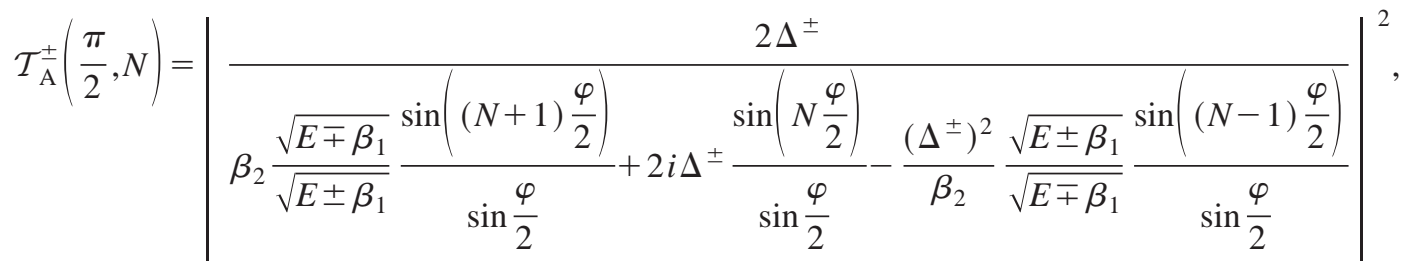

with $\quad \cos ^{2}(\varphi / 2)=\left(1 / 4 \beta_{2}^{2}\right)\left(E^{2}-\beta_{1}^{2}\right)=\cos ^{2}\left(\varphi_{1} / 2\right)$

$=\cos ^{2}\left(\varphi_{2} / 2\right)$.

For the transmission around the Fermi level, the term $\mathcal{T}_{\mathrm{A}}(0, N)$ is the most important, since the Hamiltonian $\mathcal{H}_{\mathrm{A}}(0)$ is the only one with eigenvalues close to energy zero. If we ignore curvature effects, $\mathcal{T}_{\mathrm{A}}(0, N)$ at the Fermi level is equal to

$$
\begin{aligned}
\mathcal{T}_{\mathrm{A}}(0, N) & =\frac{4}{4+\left(\frac{\Delta^{+}}{\beta}\right)^{2}}+\frac{4}{4+\left(\frac{\Delta^{-}}{\beta}\right)^{2}} \\
& \approx 2-\frac{1}{2} \frac{\Delta_{1}^{2}+\Delta_{2}^{2}}{\beta^{2}}, \quad \text { for } N=3 q-1, \\
\mathcal{T}_{\mathrm{A}}(0, N) & =\frac{4}{\left(\frac{\beta}{\Delta^{+}}+\frac{\Delta^{+}}{\beta}\right)^{2}}+\frac{4}{\left(\frac{\beta}{\Delta^{-}}+\frac{\Delta^{-}}{\beta}\right)^{2}} \\
& \approx 8 \frac{\Delta_{1}^{2}+\Delta_{2}^{2}}{\beta^{2}}, \quad \text { for } N=3 q,
\end{aligned}
$$

$$
\begin{aligned}
\mathcal{T}_{\mathrm{A}}(0, N) & =\frac{4}{4+\left(\frac{\beta}{\Delta^{+}}\right)^{2}}+\frac{4}{4+\left(\frac{\beta}{\Delta^{-}}\right)^{2}} \\
& \approx 8 \frac{\Delta_{1}^{2}+\Delta_{2}^{2}}{\beta^{2}}, \quad \text { for } N=3 q+1,
\end{aligned}
$$

with $q$ an integer. We have a maximum in the first case, a minimum in the other two cases. The approximations are for low coupling: i.e., $\Delta_{1} \rightarrow 0$ and $\Delta_{2} \rightarrow 0$. So whenever the number of rings is equal to $3 q-1$, the transmission peaks near the value 2 , a feature numerically confirmed by Orlikowski et al. ${ }^{16}$ using a simple tight-binding model with symmetric hopping parameters. These authors refer to this class of tubes as on-resonance devices. Note also that the transmission at the Fermi level for the three classes of tubes is not dependent on the length of the nanotube, as opposed to the zigzag tubes considered above.

With the introduction of curvature-i.e., leaving the isotropic model used by Orlikowski et al. - the picture becomes entirely different: the transmission at the Fermi level of $N$ $=3 q-1, N=3 q$, and $N=3 q+1$ tubes versus nanotube 
length as shown in Fig. 5(b) shows a peaked structure that repeats periodically. From Eq. (94), imposing $E=0$, and rewritten to the variable $\varphi_{1}=\varphi_{2}=4 \pi / 3+\Delta \varphi_{F}$, the two components of $\mathcal{T}_{\mathrm{A}}(0, N)$ become equal to

$$
\mathcal{T}_{\mathrm{A}}^{ \pm}(0, N)=\left|\frac{2 \Delta^{ \pm} \sin \left(\frac{2 \pi}{3}+\frac{\Delta \varphi_{F}}{2}\right)}{\beta_{2} \sin \left(\frac{(N+1) \Delta \varphi_{F}}{2}+\omega\right)+2 i \Delta^{ \pm} \sin \left(\frac{N \Delta \varphi_{F}}{2}-\frac{2 \pi}{3}+\omega\right)-\frac{\left(\Delta^{ \pm}\right)^{2}}{\beta_{2}} \sin \left(\frac{(N-1) \Delta \varphi_{F}}{2}-\frac{4 \pi}{3}+\omega\right)}\right|^{2},
$$

with $\omega$ equal to

$$
\begin{aligned}
& \omega=0, \quad \text { for } N=3 q-1, \\
& \omega=\frac{2 \pi}{3}, \quad \text { for } N=3 q, \\
& \omega=\frac{4 \pi}{3}, \quad \text { for } N=3 q+1 .
\end{aligned}
$$

So when $N$ is treated as a continuous variable, $\mathcal{T}_{\mathrm{A}}(0,3 q$ $-1), \mathcal{T}_{\mathrm{A}}(0,3 q), \mathcal{T}_{\mathrm{A}}(0,3 q+1)$ versus $N$ give rise to oscillating curves with period $2 \pi / \Delta \varphi_{F}$. They are all identical except for the shift by $\pm 2 \pi / 3 \Delta \varphi_{F}$ with respect to each other. A similar behavior of the transmission was found in sidecontacted infinite armchair nanotubes. ${ }^{28}$ In Fig. 5(b) the transmission is plotted. It is clear that the transmission at energy zero will peak whenever

$$
\frac{\sin \left((N+1) \frac{\varphi}{2}\right)}{\sin \frac{\varphi}{2}}=0
$$

and this leads to an identical condition as for a minimal HOMO-LUMO gap, which is derived in Appendix B. Conversely, the transmission will be minimal whenever the HOMO-LUMO gap is maximal. This is indeed the case when comparing Figs. 5(a) and 5(b): the same period $2 \pi / \Delta \varphi_{F}$ is encountered for the three classes of tubes. So instead of the condition that on-resonance tubes have the length $N=3 q-1$, as proposed by Orlikowski et al., ${ }^{16}$ the inclusion of curvature gives a fundamentally different condition. The value of the maxima of transmission for all kinds of tubes is then close to

$$
\begin{aligned}
& \frac{4}{4+\left(\frac{\beta_{1} \Delta^{+}}{\beta_{2}^{2}}\right)^{2}}+\frac{4}{4+\left(\frac{\beta_{1} \Delta^{-}}{\beta_{2}^{2}}\right)^{2}} \\
& \approx 2-\frac{1}{2} \frac{\beta_{1}^{2}}{\beta_{2}^{4}}\left(\Delta_{1}^{2}+\Delta_{2}^{2}\right) \approx 2-\frac{1}{2} \frac{\Delta_{1}^{2}+\Delta_{2}^{2}}{\beta^{2}} .
\end{aligned}
$$

The value near the minima is

$$
\begin{gathered}
\frac{4-\frac{\beta_{1}^{2}}{\beta_{2}^{2}}}{2+\frac{\beta_{2}^{2}}{\left(\Delta^{+}\right)^{2}}+\frac{\left(\Delta^{+}\right)^{2}}{\beta_{2}^{2}}\left(1-\frac{1}{2} \frac{\beta_{1}^{2}}{\beta_{2}^{2}}\right)^{2}} \\
+\frac{4-\frac{\beta_{1}^{2}}{\beta_{2}^{2}}}{2+\frac{\beta_{2}^{2}}{\left(\Delta^{-}\right)^{2}}+\frac{\left(\Delta^{-}\right)^{2}}{\beta_{2}^{2}}\left(1-\frac{1}{2} \frac{\beta_{1}^{2}}{\beta_{2}^{2}}\right)^{2}} \\
\approx 2\left(4-\frac{\beta_{1}^{2}}{\beta_{2}^{2}}\right) \frac{\Delta_{1}^{2}+\Delta_{2}^{2}}{\beta_{2}^{2}} \approx 6 \frac{\Delta_{1}^{2}+\Delta_{2}^{2}}{\beta^{2}} .
\end{gathered}
$$

The value of the minima does not depend on length, although the HOMO-LUMO gap decreases with length.

\section{CONCLUSIONS}

In this work we have worked out the transfer matrix method for the case of finite nanotubes with zigzag or armchair geometries. The method provides us with a powerful analytical tool to obtain eigenvectors and eigenfunctions, and from there we can infer all aspects of the transmission function. The nonsymmetric hopping parameters, which are also important to describe oscillation effects of the transmission in tip-infinite-nanotube-tip configurations, ${ }^{28}$ are necessary for a correct description of the wave functions and the transmission coefficients of finite nanotubes. In the case of zigzag tubes, the effect of different open ends is revealed: tubes with hydrogen ends on one edge and methylene ends on the other have a twice as large band gap as tubes with identical ends [Eqs. (49) and (50)]. Furthermore, the energy of their edge states is zero, irrespective of the rotational symmetry parameter $\lambda$ of their wave function (37). This is in contrast with the edge states of zigzag tubes with identical ends, were the energy is slightly dependent on $\lambda$, Eqs. (44).

The inclusion of curvature is necessary to explain the band-gap behavior of armchair tubes: at shorter lengths, we have $\Delta E_{N=3 q-1}=2\left|\beta_{1}-\beta_{2}\right|<\Delta E_{N=3 q}<\Delta E_{N=3 q+1}$, but at a certain length $\Delta E_{N=3 q}$ becomes smaller than $\Delta E_{N=3 q-1}$, at twice this length the same happens for $\Delta E_{N=3 q+1}$, and at three times this length $\Delta E_{N=3 q-1}$ starts decreasing, Eq. (65). This explains numerical calculations in Refs. 11 and 14. For larger lengths, a damped oscillatory behavior of the HOMOLUMO gap is encountered.

The difference in the transmission at the Fermi level between armchair and zigzag tubes can be explained by the different nature of the wave functions at this energy. The 
wave functions around the Fermi level for zigzag tubes are decaying, making it harder for the electron to reach the other side when the length of the tube is increased. For the armchair tube, on the other hand, there exist nondecaying, delocalized wave functions around the Fermi level that can carry the electron from lead to lead. Inclusion of curvature leads to an oscillatory behavior of this transmission that is related to the HOMO-LUMO gap oscillation and is very different from the behavior found by Orlikowski et al. ${ }^{16}$ where no curvature was included.

\section{ACKNOWLEDGMENTS}

Financial support from the Flemish government through the Concerted Action Scheme and from the Science Fund FWO-Vlaanderen is gratefully acknowledged.

\section{APPENDIX A: RELATION BETWEEN GREEN'S FUNCTION AND TRANSFER MATRIX}

We prove here relationship (14) between the transfer matrix and the Green's function. Consider the following tridiagonal block matrix:

$\left(E I-\mathcal{H}^{(N)}\right)=\left(\begin{array}{cccc}E I-H_{1} & -L_{1} & 0 & 0 \\ -L_{1}^{\dagger} & E I-H_{2} & \cdots & \vdots \\ \vdots & & & -L_{N-1} \\ 0 & \cdots & -L_{N-1}^{\dagger} & E I-H_{N}\end{array}\right)$.

We want to prove the following relationship:

$$
G_{1 N}^{(n)}=\left(T_{11}^{(n)}\right)^{-1},
$$

where $T^{(n)}$ is the transfer matrix and $G^{(n)}$ the Green's function matrix of the matrix $\left(E I-\mathcal{H}^{(n)}\right)$ which is a submatrix of $\left(E I-\mathcal{H}^{(N)}\right)$ with matrix blocks running from 1 to $n$. It is a trivial task to prove that, for $N=1$,

$$
\left(G_{11}^{(1)}\right)^{-1}=E I-H_{1}=T_{11}^{(1)} .
$$

From the definition of the transfer matrix one obtains

$$
\begin{aligned}
\left\{T_{n} \cdots T_{1}\right\}_{11}= & L_{n}^{-1}\left(E-H_{n}\right)\left\{T_{n-1} \cdots T_{1}\right\}_{11} \\
& -L_{n}^{-1} L_{n-1}^{\dagger}\left\{T_{n-2} \cdots T_{1}\right\}_{11} .
\end{aligned}
$$

Of course, $T_{11}^{(N)}=\left\{T_{N} \cdots T_{1}\right\}_{11}$. Further $L_{N}=I$ since it is no part of $\mathcal{H}^{(N)}$ and $L_{n}^{-1} T_{11}^{(n)}=\left\{T_{n} \cdots T_{1}\right\}_{11}$ since $L_{n}$ is no part of $\mathcal{H}^{(n)}$ :

$$
T_{11}^{(N)}=\left(E-H_{N}\right) L_{N-1}^{-1} T_{11}^{(N-1)}-L_{N-1}^{\dagger} L_{N-2}^{-1} T_{11}^{(N-2)} .
$$

On the other hand, we have

$$
\begin{aligned}
G^{(N)} & =\left(\begin{array}{cccc}
E I-H_{1} & -L_{1} & 0 & 0 \\
-L_{1}^{\dagger} & E I-H_{2} & \cdots & \vdots \\
\vdots & & & -L_{N-1} \\
0 & \cdots & -L_{N-1}^{\dagger} & E I-H_{N}
\end{array}\right)^{-1} \\
& =\left(\begin{array}{cc}
G_{A} & G_{1 N}^{(N)} \\
G_{C} & G_{B}
\end{array}\right) .
\end{aligned}
$$

From Eq. (A6) we can write

$$
\begin{aligned}
& G_{A}\left(\begin{array}{c}
0 \\
\vdots \\
-L_{N-1}
\end{array}\right)+G_{1 N}^{(N)}\left(E I-H_{N}\right)=\mathbf{0}, \\
& G_{A}\left(E I-\mathcal{H}^{(N-1)}\right)+G_{1 N}^{(N)}\left(\begin{array}{lll}
0 & \cdots & -L_{N-1}^{\dagger}
\end{array}\right)=\left(\begin{array}{lll}
I & \cdots & 0
\end{array}\right) .
\end{aligned}
$$

After elimination of $G_{A}$, one obtains

$$
G_{1 N}^{(N)}=G_{1 N-1}^{(N-1)} L_{N-1}\left(E I-H_{N}-L_{N-1}^{\dagger} G_{N-1 N-1}^{(N-1)} L_{N-1}\right)^{-1},
$$

and in a similar way, one can prove $\mathrm{e}^{19}$

$$
G_{N N}^{(N)}=\left(E I-H_{N}-L_{N-1}^{\dagger} G_{N-1 N-1}^{(N-1)} L_{N-1}\right)^{-1} .
$$

From Eqs. (A8) and (A9) we obtain

$$
\begin{aligned}
G_{1 N}^{(N)}= & G_{1 N-1}^{(N-1)} L_{N-1} G_{N N}^{(N)} \\
& \Rightarrow G_{N N}^{(N)}\left(G_{1 N}^{(N)}\right)^{-1}=L_{N-1}^{-1}\left(G_{1 N-1}^{(N-1)}\right)^{-1} .
\end{aligned}
$$

Inverting Eq. (A8) yields

$$
\begin{aligned}
\left(G_{1 N}^{(N)}\right)^{-1}= & \left(E I-H_{N}-L_{N-1}^{\dagger} G_{N-1 N-1}^{(N-1)} L_{N-1}\right) \\
& \times L_{N-1}^{-1}\left(G_{1 N-1}^{(N-1)}\right)^{-1} \\
= & \left(E I-H_{N}\right) L_{N-1}^{-1}\left(G_{1 N-1}^{(N-1)}\right)^{-1} \\
& -L_{N-1}^{\dagger} G_{N-1 N-1}^{(N-1)}\left(G_{1 N-1}^{(N-1)}\right)^{-1} \\
= & \left(E I-H_{N}\right) L_{N-1}^{-1}\left(G_{1 N-1}^{(N-1)}\right)^{-1} \\
& -L_{N-1}^{\dagger} L_{N-2}^{-1}\left(G_{1 N-2}^{(N-2)}\right)^{-1} .
\end{aligned}
$$

This relationship is identical to Eq. (A5) if one substitutes $\left(G_{1 N}^{(n)}\right)^{-1}$ with $T_{11}^{(n)}$ and since $\left(G_{1 N}^{(1)}\right)^{-1}=T_{11}^{(1)}$, we have proved that $\left(G_{1 N}^{(n)}\right)^{-1}=T_{11}^{(n)}$ for every $n$.

\section{APPENDIX B: HOMO-LUMO GAP OF ARMCHAIR TUBES}

We construct an analytical formula that generates the HOMO-LUMO gap for armchair tubes. It will have the following form:

$$
\Delta E=2\left|\beta_{1}+2 \beta_{2} \cos \left(\frac{r \pi}{N+1}\right)\right|,
$$

where $r$ is an integer, chosen so that it minimizes $\Delta E$. The Fermi point $\varphi_{F}$ is defined as

$$
\Delta E=2\left|\beta_{1}+2 \beta_{2} \cos \left(\frac{\varphi_{F}}{2}\right)\right|=0 .
$$

In the case of no curvature, the Fermi point $\varphi_{F}$ is equal to $4 \pi / 3$. When $N=3 q-1$, this point is always an allowed point-namely, for $r=2 q$ - and we have a zero band gap. When $N=3 q$ or $N=3 q+1$, the closest point to $4 \pi / 3$ is for $2 q+1$. Filling in in Eq. (B1) leads to Eq. (64).

If we want to include curvature, the case is more complicated. Let us substitute $r$ with $\widetilde{r}-1+\varkappa$ and define $\Delta \varphi_{\varkappa}$ :

$$
\Delta \varphi_{\varkappa}=\frac{2 r \pi}{N+1}-\frac{4 \pi}{3}=\frac{2 \pi}{3} \frac{3(\widetilde{r}-1+\varkappa)-2(N+1)}{N+1} .
$$

In this way the lowest positive value of $\Delta \varphi_{\varkappa}$ is for $\varkappa=1$. The value of $\Delta \varphi_{\varkappa}$ for the three kinds of tubes is then equal to 


$$
\begin{aligned}
& \Delta \varphi_{\varkappa}=2 \pi \frac{\varkappa}{N+1}, \quad \text { for } N+1=3 q, \\
& \Delta \varphi_{\varkappa}=2 \pi \frac{\varkappa-\frac{2}{3}}{N+1}, \quad \text { for } N+1=3 q+1, \\
& \Delta \varphi_{\varkappa}=2 \pi \frac{\varkappa-\frac{1}{3}}{N+1}, \quad \text { for } N+1=3 q+2 .
\end{aligned}
$$

Because of curvature, the Fermi point $\varphi_{F}$ moves away from the point $4 \pi / 3$ :

$$
\varphi_{F}=\frac{4 \pi}{3}+\Delta \varphi_{F},
$$

with

$$
\Delta \varphi_{F}=-\frac{4}{3} \pi+2 \arccos \left(-\frac{\beta_{1}}{2 \beta_{2}}\right) .
$$

If $\left|\beta_{1}\right|>\left|\beta_{2}\right|$, like we suppose, then $\Delta \varphi_{F}>0$. Close to the Fermi level we can make the approximation that

$$
E\left(\varphi_{F}-\Delta x\right) \approx E\left(\varphi_{F}+\Delta x\right) \approx \sigma\left|\frac{1}{2} \sqrt{4 \beta_{2}^{2}-\beta_{1}^{2}}\right||\Delta x|,
$$

so the band gap is determined by $\varkappa$ minimizing the difference $\Delta \varphi_{F}-\Delta \varphi_{\varkappa}$, whether it is positive or negative. In other words,

$$
\begin{aligned}
& N=3 q-1 \Rightarrow \varkappa=\operatorname{Int}\left[\frac{\Delta \varphi_{F}}{2 \pi}(N+1)\right], \\
& N=3 q \Rightarrow \varkappa=\operatorname{Int}\left[\frac{\Delta \varphi_{F}}{2 \pi}(N+1)+\frac{2}{3}\right], \\
& N=3 q+1 \Rightarrow \varkappa=\operatorname{Int}\left[\frac{\Delta \varphi_{F}}{2 \pi}(N+1)+\frac{1}{3}\right],
\end{aligned}
$$

with $\operatorname{Int}[x]$ the integer closest to $x$. This gives, for the band gap,

$$
\begin{aligned}
\Delta E= & 2 \mid \beta_{1}+2 \beta_{2} \cos \left\{\frac{2 \pi}{3}+\frac{\pi}{N+1}\right. \\
& \left.\times\left(\operatorname{Int}\left[\frac{\Delta \varphi_{F}}{2 \pi}(N+1)+h\right]-h\right)\right\} \mid,
\end{aligned}
$$

with $h$ equal to

$$
\begin{aligned}
& h=0, \quad \text { for } N=3 q-1, \\
& h=\frac{2}{3}, \quad \text { for } N=3 q, \\
& h=\frac{1}{3}, \quad \text { for } N=3 q+1 .
\end{aligned}
$$

\section{APPENDIX C: DETERMINANTS, WAVE FUNCTIONS AND TRANSMISSION COEFFICIENTS}

Here we show how to deduce determinants, wave functions, and transmission coefficients for zigzag and armchair nanotubes.

\section{Zigzag tubes}

Because we assume that the leads do not lower the high rotational symmetry of zigzag tubes, the matrices $\Sigma_{L}$ and $\Sigma_{R}$ become completely diagonalized. Following Eqs. (23) we obtain

$$
\begin{aligned}
& \Sigma_{11}(\lambda)=\frac{1}{n} \sum_{k=0}^{n-1} e^{2 i \lambda k}\left\langle\chi_{01}\left|\Sigma_{L}\right| \chi_{k 1}\right\rangle \\
& \Sigma_{N N}(\lambda)=\frac{1}{n} \sum_{k=0}^{n-1} e^{2 i \lambda k}\left\langle\chi_{0 N}\left|\Sigma_{L}\right| \chi_{k N}\right\rangle .
\end{aligned}
$$

For simplicity, we take the same parameter for all diagonal elements, assume that it is constant in energy and purely imaginary, and take identical leads. So we have

$$
\Sigma_{11}(\lambda)=\Sigma_{N N}(\lambda)=-i \Delta,
$$

and find, in the case of HH or MM tubes from Eq. (24) for the matrix element $T_{11}$,

$$
T_{11}=b_{2} \frac{\sin \frac{N+2}{2} \varphi+\frac{b_{2}}{b_{1}} \sin \frac{N}{2} \varphi}{\sin \varphi}+2 i \Delta \frac{E}{b_{1}} \frac{\sin \frac{N}{2} \varphi}{\sin \varphi}
$$

$$
\begin{aligned}
& -\frac{\Delta^{2}}{b_{1}} \frac{\sin \frac{N}{2} \varphi+\frac{b_{1}}{b_{2}} \sin \frac{N-2}{2} \varphi}{\sin \varphi} \\
= & \left(b_{1} b_{2}\right)^{-N / 2} b_{2} \widetilde{D}_{\mathrm{Z}}^{\mathrm{XX}}(\lambda, N) \\
= & \left(b_{1} b_{2}\right)^{-N / 2} b_{2}\left[D_{\mathrm{Z}}^{\mathrm{XX}}(\lambda, N)+2 i \Delta D_{\mathrm{Z}}^{\mathrm{XY}}(\lambda, N-1)\right. \\
& \left.-\Delta^{2} D_{\mathrm{Z}}^{\mathrm{YY}}(\lambda, N-2)\right],
\end{aligned}
$$

with $\mathrm{X} \neq \mathrm{Y}$, and a similar expression for HM tubes:

$$
\begin{aligned}
& T_{11}=E \frac{\sin \frac{N+1}{2} \varphi}{\sin \varphi} \\
& +i \Delta\left(\frac{2 \sin \frac{N+1}{2} \varphi+\left(\frac{b_{1}}{b_{2}}+\frac{b_{2}}{b_{1}}\right) \sin \frac{N-1}{2} \varphi}{\sin \varphi}\right) \\
& -\frac{\Delta^{2}}{b_{1}} \frac{E}{b_{2}} \frac{\sin \frac{N-1}{2} \varphi}{\sin \varphi} \\
& =\left(b_{1} b_{2}\right)^{-(N-1) / 2} \widetilde{D}_{\mathrm{Z}}^{\mathrm{XY}}(\lambda, N) \\
& =\left(b_{1} b_{2}\right)^{-(N-1) / 2}\left\{D_{\mathrm{Z}}^{\mathrm{XY}}(\lambda, N)+i \Delta\left[D_{\mathrm{Z}}^{\mathrm{XX}}(\lambda, N-1)\right.\right. \\
& \left.\left.+D_{\mathrm{Z}}^{\mathrm{YY}}(\lambda, N-1)\right]-\Delta^{2} D_{\mathrm{Z}}^{\mathrm{XY}}(\lambda, N-2)\right\} \text {. }
\end{aligned}
$$

By setting the coupling $\Delta$ to zero and via Eq. (4) we get the determinant of an isolated zigzag tube in Eqs. (35). The coefficients of the wave function can be found via Eqs. (2). The transmission is equal to

$$
\mathcal{T}_{\mathrm{Z}}(\lambda, N)=\operatorname{Tr}\left(Q_{\mathrm{Z}}(\lambda, N)\right)=2 \Delta T_{11}^{-1} 2 \Delta\left(T_{11}^{\dagger}\right)^{-1}=\frac{4 \Delta^{2}}{\left|T_{11}\right|^{2}},
$$


and via Eq. (C3) or (C4) in the case of a XX or HM tube, respectively, one obtains Eqs. (79).

\section{Armchair tubes}

Because we assume that the leads do not lower the high rotational symmetry of zigzag tubes, the matrices $\Sigma_{L}$ and $\Sigma_{R}$ become block-diagonalized into $2 \times 2$ matrices following Eqs. (23):

$$
\left(\Sigma_{11}(\lambda)\right)_{r r^{\prime}}=\frac{1}{n} \sum_{k=0}^{n-1} e^{2 i \lambda k}\left\langle\chi_{r 01}\left|\Sigma_{L}\right| \chi_{r^{\prime} k 1}\right\rangle,
$$

$$
\left(\Sigma_{N N}(\lambda)\right)_{r r^{\prime}}=\frac{1}{n} \sum_{k=0}^{n-1} e^{2 i \lambda k}\left\langle\chi_{r 0 N}\left|\Sigma_{L}\right| \chi_{r^{\prime} k N}\right\rangle,
$$

where $r$ and $r^{\prime}$ can be 1 or 2. For simplicity, we take each matrix block identical and purely imaginary:

$$
\Sigma_{11}(\lambda)=\Sigma_{N N}(\lambda)=\left(\begin{array}{cc}
-i \Delta_{1} & -i \Delta_{2} \\
-i \Delta_{2} & -i \Delta_{1}
\end{array}\right)
$$

We find from Eq. (24), for the matrix block $T_{11}$,

$$
\begin{aligned}
T_{11}= & -\beta_{2}\left(\begin{array}{cc}
e^{i \lambda} S(N+1) & R_{2}(N+1) \\
R_{1}(N+1) & e^{-i \lambda} S(N+1)
\end{array}\right)+\left(\begin{array}{cc}
i \Delta_{1} & i \Delta_{2} \\
i \Delta_{2} & i \Delta_{1}
\end{array}\right)\left(\begin{array}{cc}
\Delta e^{i \lambda}\left[R_{1}(N)+R_{2}(N)\right] & 2 S(N) \\
2 S(N) & e^{-i \lambda}\left[R_{1}(N)+R_{2}(N)\right]
\end{array}\right) \\
& +\frac{1}{\beta_{2}}\left(\begin{array}{cc}
\Delta_{1}^{2}+\Delta_{2}^{2} & 2 \Delta_{1} \Delta_{2} \\
2 \Delta_{1} \Delta_{2} & \Delta_{1}^{2}+\Delta_{2}^{2}
\end{array}\right)\left(\begin{array}{cc}
e^{-i \lambda} S(N-1) & R_{1}(N-1) \\
R_{2}(N-1) & e^{i \lambda} S(N-1)
\end{array}\right), \text { for } N \text { even, } \\
T_{11}= & \beta_{2}\left(\begin{array}{cc}
S(N+1) & e^{-i \lambda} R_{2}(N+1) \\
e^{i \lambda} R_{1}(N+1) & S(N+1)
\end{array}\right)-\left(\begin{array}{cc}
i \Delta_{1} & i \Delta_{2} \\
i \Delta_{2} & i \Delta_{1}
\end{array}\right)\left(\begin{array}{cc}
R_{1}(N)+R_{2}(N) & 2 e^{-i \lambda} S(N) \\
2 e^{i \lambda} S(N) & R_{1}(N)+R_{2}(N)
\end{array}\right)-\frac{1}{\beta_{2}}\left(\begin{array}{cc}
\Delta_{1}^{2}+\Delta_{2}^{2} & 2 \Delta_{1} \Delta_{2} \\
2 \Delta_{1} \Delta_{2} & \Delta_{1}^{2}+\Delta_{2}^{2}
\end{array}\right) \\
& \times\left(\begin{array}{cc}
S(N-1) & e^{-i \lambda} R_{1}(N-1) \\
e^{i \lambda} R_{2}(N-1) & S(N-1)
\end{array}\right), \text { for } N \text { odd, }
\end{aligned}
$$

Putting $\Delta_{1}=\Delta_{2}=0$ and using Eq. (4) gives the determinant of an isolated armchair tube. The coefficients of the wave function can be found via Eqs. (2).

Using Eqs. (12) and (14) the transmission is equal to

$$
\begin{aligned}
\mathcal{T}_{\mathrm{A}}(\lambda, N) & =\operatorname{Tr}\left[Q_{\mathrm{A}}(\lambda, N)\right]=\operatorname{Tr}\left[\left(\begin{array}{cc}
2 \Delta_{1} & 2 \Delta_{2} \\
2 \Delta_{2} & 2 \Delta_{1}
\end{array}\right)\left(\begin{array}{cc}
a & b \\
c & d
\end{array}\right)^{-1}\left(\begin{array}{cc}
2 \Delta_{1} & 2 \Delta_{2} \\
2 \Delta_{2} & 2 \Delta_{1}
\end{array}\right)\left(\begin{array}{cc}
a^{*} & c^{*} \\
b^{*} & d^{*}
\end{array}\right)^{-1}\right] \\
& =\frac{4\left\{\Delta_{1}^{2}\left(|a|^{2}+|c|^{2}+|b|^{2}+|d|^{2}\right)+\Delta_{2}^{2}\left(a d^{*}+b^{*} c+\text { c.c. }\right)-\Delta_{1} \Delta_{2}\left[\left(a^{*}+d^{*}\right)(b+c)+\text { c.c. }\right]\right\}}{|a d-b c|^{2}},
\end{aligned}
$$

where $T_{11}$ was rewritten as

$$
T_{11}=\left(\begin{array}{ll}
a & b \\
c & d
\end{array}\right) .
$$

It is possible to get the contributions of the individual channels by diagonalizing $Q_{\mathrm{A}}(\lambda, N)$ but we have not done it here, except in the case of $\lambda=0$ and $\lambda=\pi / 2$ where we can apply mirror symmetry for a complete diagonalization.

${ }^{1}$ S. Iijima, Nature (London) 354, 56 (1991).

${ }^{2}$ Carbon Nanotubes [Acc. Chem. Res. 35, 1 2002].

${ }^{3}$ R. A. Jishi, J. Bragin, and L. Lou, Phys. Rev. B 59, 9862 (1999).

${ }^{4}$ A. Rubio, D. Sanchez-Portal, E. Artacho, P. Ordejon, and J. M. Soler, Phys. Rev. Lett. 82, 3520 (1999).

${ }^{5}$ J. Li, Y. Zhang, and M. Zhang, Chem. Phys. Lett. 364, 328 (2002).

${ }^{6}$ D. J. Klein, Chem. Phys. Lett. 217, 261 (1994).

${ }^{7}$ M. Fujita, K. Wakabayashi, K. Nakada, and K. Kusakabe, J. Phys. Soc. Jpn. 65, 1920 (1996).

${ }^{8}$ K. Nakada, M. Fujita, G. Dresselhaus, and M. S. Dresselhaus, Phys. Rev. B 54, 17954 (1996).

${ }^{9}$ K. Wakabayashi, M. Fujita, H. Ajiki, and M. Sigrist, Phys. Rev. B 59, 8271 (1999).

${ }^{10}$ G. Treboux, P. Lapstun, and K. Silverbrook, Chem. Phys. Lett. 302, 60 (1999).
${ }^{11}$ A. Rochefort, D. R. Salahub, and P. Avouris, J. Phys. Chem. B 103, 641 (1999).

${ }^{12}$ T. Sato, M. Tanaka, and T. Yamabe, Synth. Met. 103, 2525 (1999).

${ }^{13}$ T. Yamabe, M. Imade, M. Tanaka, and T. Sato, Synth. Met. 117, 61 (2001).

${ }^{14}$ L. Liu, C. Jayanthi, H. Guo, and S. Wu, Phys. Rev. B 64, 033414 (2001).

${ }^{15}$ H.-Y. Zhu, D. J. Klein, T. G. Schmalz, A. Rubio, and N. March, J. Phys. Chem. Solids 59, 417 (1998).

${ }^{16}$ D. Orlikowski, H. Mehrez, J. Taylor, H. Guo, and J. Wang, Phys. Rev. B 63, 155412 (2001).

${ }^{17}$ L. Molinari, J. Phys. A 30, 983 (1997).

${ }^{18}$ G. Biczó, in Recent Developments in Condensed Matter Physics, edited by J. T. Devreese, L. F. Lemmens, V. E. V. Doren, and J. V. Royen (Plenum, New York, 1981), Vol. 2, pp. 341-351.

${ }^{19}$ S. Datta, Electronic Transport in Mesoscopic Systems (Cambridge University Press, Cambridge, England, 1997). 
${ }^{20}$ D. S. Fisher and P. A. Lee, Phys. Rev. B 23, 6851 (1981).

${ }^{21}$ J. Heurich, J. C. Cuevas, W. Wenzel, and G. Schön, Phys. Rev. Lett. 88, 256803 (2002)

${ }^{22}$ J. J. Palacios, E. Louis, A. J. Pérez-Jiménez, E. S. Fabian, and J. A. Vergés, Nanotechnology 13, 378 (2002).

${ }^{23}$ W. Tian et al., J. Chem. Phys. 109, 2874 (1998).
${ }^{24}$ K. Wakabayashi, Phys. Rev. B 64, 125428 (2001).

${ }^{25}$ A. Ceulemans, L. F. Chibotaru, S. A. Bovin, and P. W. Fowler, J. Chem. Phys. 112, 4271 (2000).

${ }^{26}$ V. Mujica, M. Kemp, and M. A. Ratner, J. Chem. Phys. 101, 6849 (1994).

${ }^{27}$ F. J. Dyson, Phys. Rev. 75, 486 (1949).

${ }^{28}$ L. F. Chibotaru, S. Compernolle, and A. Ceulemans (unpublished). 
The Journal of Chemical Physics is copyrighted by the American Institute of Physics (AIP). Redistribution of journal material is subject to the AIP online journal license and/or AIP copyright. For more information, see http:/ojps.aip.org/jcpo/jcpcr/jsp Copyright of Journal of Chemical Physics is the property of American Institute of Physics and its content may not be copied or emailed to multiple sites or posted to a listserv without the copyright holder's express written permission. However, users may print, download, or email articles for individual use. 